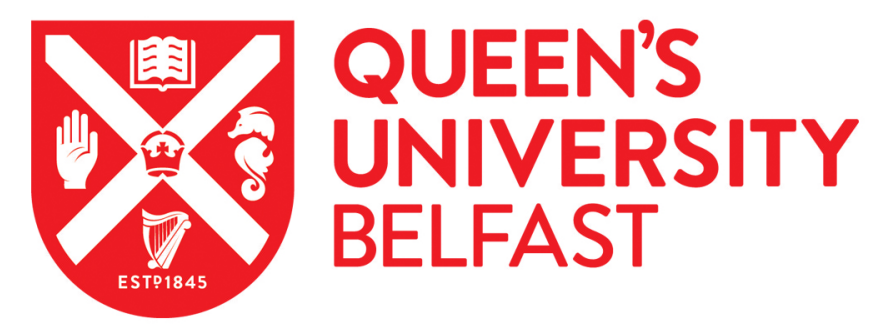

\title{
Nonequilibrium processes from generalized Langevin equations: Realistic nanoscale systems connected to two thermal baths
}

Ness, H., Genina, A., Stella, L., Lorenz, C. D., \& Kantorovich, L. (2016). Nonequilibrium processes from generalized Langevin equations: Realistic nanoscale systems connected to two thermal baths. Physical Review B (Condensed Matter), 93(17), [174303]. https://doi.org/10.1103/PhysRevB.93.174303

Published in:

Physical Review B (Condensed Matter)

Document Version:

Publisher's PDF, also known as Version of record

Queen's University Belfast - Research Portal:

Link to publication record in Queen's University Belfast Research Portal

Publisher rights

(C) 2016 American Physical Society

\section{General rights}

Copyright for the publications made accessible via the Queen's University Belfast Research Portal is retained by the author(s) and / or other copyright owners and it is a condition of accessing these publications that users recognise and abide by the legal requirements associated with these rights.

Take down policy

The Research Portal is Queen's institutional repository that provides access to Queen's research output. Every effort has been made to ensure that content in the Research Portal does not infringe any person's rights, or applicable UK laws. If you discover content in the Research Portal that you believe breaches copyright or violates any law, please contact openaccess@qub.ac.uk. 


\title{
Nonequilibrium processes from generalized Langevin equations: Realistic nanoscale systems connected to two thermal baths
}

\author{
H. Ness $,{ }^{1},{ }^{*}$ A. Genina, ${ }^{1}$ L. Stella, ${ }^{2}$ C. D. Lorenz, ${ }^{1}$ and L. Kantorovich ${ }^{1}$ \\ ${ }^{1}$ Department of Physics, Faculty of Natural and Mathematical Sciences, King's College London, Strand, London WC2R 2LS, United Kingdom \\ ${ }^{2}$ Atomistic Simulation Centre, School of Mathematics and Physics, Queen's University Belfast, \\ University Road, Belfast BT7 1NN, Northern Ireland, United Kingdom
}

(Received 27 November 2015; revised manuscript received 22 April 2016; published 4 May 2016)

\begin{abstract}
We extend the generalized Langevin equation (GLE) method [L. Stella, C. D. Lorenz, and L. Kantorovich, Phys. Rev. B 89, 134303 (2014)] to model a central classical region connected to two realistic thermal baths at two different temperatures. In such nonequilibrium conditions a heat flow is established, via the central system, in between the two baths. The GLE-2B (GLE two baths) scheme permits us to have a realistic description of both the dissipative central system and its surrounding baths. Following the original GLE approach, the extended Langevin dynamics scheme is modified to take into account two sets of auxiliary degrees of freedom corresponding to the mapping of the vibrational properties of each bath. These auxiliary variables are then used to solve the non-Markovian dissipative dynamics of the central region. The resulting algorithm is used to study a model of a short Al nanowire connected to two baths. The results of the simulations using the GLE-2B approach are compared to the results of other simulations that were carried out using standard thermostatting approaches (based on Markovian Langevin and Nosé-Hoover thermostats). We concentrate on the steady-state regime and study the establishment of a local temperature profile within the system. The conditions for obtaining a flat profile or a temperature gradient are examined in detail, in agreement with earlier studies. The results show that the GLE-2B approach is able to treat, within a single scheme, two widely different thermal transport regimes, i.e., ballistic systems, with no temperature gradient, and diffusive systems with a temperature gradient.
\end{abstract}

DOI: 10.1103/PhysRevB.93.174303

\section{INTRODUCTION}

Nanoscale devices and materials are becoming increasingly important in the development of novel technologies. In most applications of these new nanotechnologies, the central system is part of a more complex setup where driving forces are present to establish heat and/or particle flows. The understanding of the corresponding nonequilibrium properties is of utmost importance. This is especially true when one considers potential applications based on the thermal conductivity of materials [1-8] and the heat transport within nanodevices [9-23].

Being able to describe the dynamics and dissipation of such nanoscale atomic systems is central for modern nanoscience. For that, one has to consider the central region of interest as an open system surrounded by a heat bath (an environment) which is in contact with the system and is kept at a given temperature. For studying the transport properties, one has to consider the proper nonequilibrium conditions; i.e., the central region is connected to two (or more) independent heat baths (kept at their own temperatures). Hence a heat flow (transient or stationary depending on the experimental conditions) is established between the two baths via the central region.

An appropriate general approach for treating this kind of system is based on the so-called generalized Langevin equation (GLE) [11,24-53]. The GLE is an equation of motion containing non-Markovian stochastic processes where the particle (point particle with mass) has a memory effect to its velocity.

\footnotetext{
*herve.ness@kcl.ac.uk
}

The GLE has been derived for a realistic system of $N$ particles coupled to a single realistic (harmonic) bath, i.e., a bath described at the atomic level [42]. The non-Markovian dynamics is obtained for the central system with Gaussian distributed random forces and a memory kernel that is exactly proportional to the random force autocorrelation function [42]. Solving the GLE for complex heterogeneous and extended systems is still a challenge. A major step towards the solution of this problem for a realistic application has been recently given [43,44,47,48,52]. In particular, an efficient and transferable algorithm has been developed in Ref. [52] to solve the GLE numerically while taking into account the two fundamental features of the GLE- a time-dependent memory kernel and the presence of a colored noise-which are absolutely essential for the description of the bath at the atomic level.

However, the previous tools have been developed for a single bath only. In order to treat properly the presence of a (transient or steady) flow of heat current through the central system, one has to take into account the proper nonequilibrium conditions. That is, one has to consider the presence of at least two baths (at their own temperatures) in contact with the central region.

The aim of the present paper is to extend the previous GLE approaches developed in Refs. [42,52,53] to the systems consisting of a central region connected to two spatially separated thermal baths. With such an approach, we can study the nonequilibrium processes in nanoscale systems by using molecular dynamics (MD) simulations. The dissipative processes are properly described since the system can exchange energy (heat) with the environment. The environment consists of the two baths whose dynamical properties are described more thoroughly than when standard thermostats 
(i.e., Langevin, Nosé-Hoover, velocity renormalization, etc.) [54-58] are used in conventional MD simulations. Note that, for a system at equilibrium, the Langevin dynamics applied only to a part of the system can be derived from the GLE assuming short-range atomic interactions and the Markovian approximation [59]. It was also shown in [59] that thermostatting only some of the degrees of freedom by using the Langevin dynamics brings the system, in equilibrium, to the corresponding canonical distribution. It can also be shown that the application of the Nosé method to only a part of the system (i.e., to a subset of atomic degrees of freedom) also performs correct thermostatting of the entire system to the target temperature.

The paper is organized as follows. In Sec. II, we generalize the methodology of Refs. [42,52,53] to the cases of two independent baths $v=1,2$ each having its temperature $T_{v}$. The generalization, called GLE-2B, includes the use of two sets of auxiliary degrees of freedom (DOFs) corresponding to each bath and their stochastic dynamics. This is performed by the use of a multivariate Markovian stochastic process for the position and momentum of the DOFs of the central region and the corresponding "position" and "momentum" of the two sets of auxiliary DOFs [52]. The resulting algorithm is explicitly described in Appendix A and has been implemented in the code LAMMPS [60] following our previous work on the GLE with a single bath [53]. In Sec. III, we consider some applications of the GLE-2B for a specific realistic system. It consists of a short $\mathrm{Al}$ nanowire connected to two $\mathrm{Al}$ baths. Each bath is represented by a set of auxiliary DOFs generated from a model solid, i.e., one half sphere of an $\mathrm{Al}$ fcc lattice. In this first application of our GLE-2B approach, we concentrate on the steady-state properties of the system. First, we consider the equilibrium condition and an artificially thermally decoupled system to perform a first validation of our methodology. Then we treat the proper nonequilibrium conditions when $T_{1} \neq T_{2}$. We also compare our results with other possible thermostatting procedures. In Sec. III B we interpret our results for the temperature profiles through the system in terms of the properties of integrable versus nonintegrable cases. In the latter case, a full temperature gradient is established in the system, while in the former case there is no temperature gradient built up in the system. In terms of transport properties, a perfect thermal conductor (a ballistic thermal system) has an infinite conductance (in the thermodynamic limit) and, therefore, there is no temperature gradient within the central part of the system. Whenever the system presents some form of thermal resistance (finite conductance), a temperature gradient exists in the system. Finally, in Sec. IV, we conclude and discuss further developments of the present study.

\section{GENERALIZATION TO THE TWO BATHS PROBLEM}

\section{A. Equations of motion for a system coupled to two baths: Embedding Newton's equations}

We consider a central system (of finite size) interacting with two independent baths $v=1,2$ [64]; see Fig. 1 for a schematic representation of the system. The corresponding classical Lagrangian is given by $\mathcal{L} \equiv \mathcal{L}_{\text {sys }}+\mathcal{L}_{\text {bath,(1) }}+\mathcal{L}_{\text {bath,(2) }}+\mathcal{L}_{\text {int }}$,

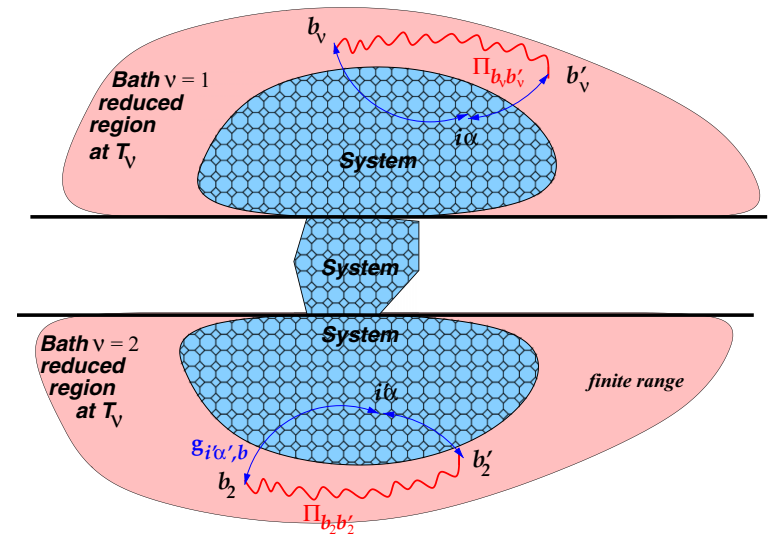

FIG. 1. Schematic representation of the system. It includes the finite size central system (in blue) where the GLE dynamics is performed, and the two bath $v=1,2$ regions at temperature $T_{v}$. Because the forces $f_{b_{v}}$ and the quantities $g_{i \alpha, b_{v}}$ are of finite range (not necessarily short ranged), one can perform the mapping of $\Pi_{b_{v} b_{v}^{\prime}}(\omega)$ on a finite region of space: the bath reduced region (in pink), one for each bath.

where

$$
\begin{gathered}
\mathcal{L}_{\text {sys }}(\mathbf{r}, \dot{\mathbf{r}})=\sum_{i \alpha} \frac{1}{2} m_{i} \dot{r}_{i \alpha}^{2}-V(\mathbf{r}), \\
\mathcal{L}_{\text {bath },(1)}\left(\mathbf{u}_{1}, \dot{\mathbf{u}}_{1}\right)=\sum_{l_{1} \gamma} \frac{1}{2} \mu_{l_{1}} \dot{u}_{l_{1} \gamma}^{2}-V_{(1)}^{\mathrm{harm}}\left(\mathbf{u}_{1}\right), \\
\mathcal{L}_{\text {int }}(\mathbf{r}, \mathbf{u})=\mathcal{L}_{\text {int },(1)}\left(\mathbf{r}, \mathbf{u}_{1}\right)+\mathcal{L}_{\text {int },(2)}\left(\mathbf{r}, \mathbf{u}_{2}\right) .
\end{gathered}
$$

The positions of the atoms, labeled $i=1,2, \ldots, N$ with mass $m_{i}$, of the central system are given by vectors $\mathbf{r}$ with components $r_{i \alpha}$ ( $\alpha$ indicating the appropriate Cartesian coordinate). $\mathcal{L}_{\text {sys }}$ is the Lagrangian of the system with potential energy $V(\mathbf{r})$. The Lagrangian $\mathcal{L}_{\text {bath,(1) }}$ describes the harmonic bath (bath $v=1$ ). The bath's atoms are labeled $l_{1}$ and have masses $\mu_{l_{1}}$. We introduce a shorthand notation for the labels of the bath degrees of freedom (DOFs) $b_{1} \equiv l_{1} \gamma$, where $\gamma$ indicates the Cartesian coordinate. The corresponding potential energy $V_{(1)}^{\text {harm }}\left(\mathbf{u}_{1}\right)$ is harmonic with respect to the displacements $u_{l_{1} \gamma}=u_{b_{1}}$ of the bath atoms from their equilibrium positions. Its expression is

$$
V_{(1)}^{\mathrm{harm}}\left(\mathbf{u}_{1}\right)=\frac{1}{2} \sum_{b_{1}, b_{1}^{\prime}} \sqrt{\mu_{l_{1}} \mu_{l_{1}^{\prime}}} u_{b_{1}} D_{b_{1}, b_{1}^{\prime}}^{(1)} u_{b_{1}^{\prime}},
$$

where $D_{b_{1}, b_{1}^{\prime}}^{(1)}$ are the elements of the dynamic matrix of the bath $v=1$. The Lagrangian $\mathcal{L}_{\text {bath,(2) }}$ for the bath $v=2$ is similar to $\mathcal{L}_{\text {bath,(1) }}$ and obtained from $\mathcal{L}_{\text {bath,(1) }}$ by swapping the bath index $1 \leftrightarrow 2$.

Finally the interaction between the central system and the baths is a linear superposition of the interaction between the system and each bath (we recall that the baths are independent). The individual contribution $\mathcal{L}_{\text {int },(v)}\left(\mathbf{r}, \mathbf{u}_{v}\right)$ is taken to be linear in the bath displacements $\mathbf{u}_{v}$ with the following expression:

$$
\mathcal{L}_{\text {int },(v)}\left(\mathbf{r}, \mathbf{u}_{v}\right)=-\sum_{b_{v}} \mu_{l_{v}} f_{b_{v}}(\mathbf{r}) u_{b_{v}} .
$$


Note that the dependence of such an interaction on the system DOFs, via $f_{b_{v}}(\mathbf{r})$, remains arbitrary.

We can now derive the equations of motion for the central system and baths DOFs from the Lagrangian, Eqs. (1)-(4), following Refs. [42,52]. We find, for the central system DOFs, that

$$
m_{i} \ddot{r}_{i \alpha}=-\frac{\partial V(\mathbf{r})}{\partial r_{i \alpha}}-\sum_{\nu=1}^{2} \sum_{b_{\nu}} \mu_{l_{\nu}} g_{i \alpha, b_{v}}(\mathbf{r}) u_{b_{v}},
$$

where $g_{i \alpha, b_{v}}(\mathbf{r})=\partial f_{b_{v}}(\mathbf{r}) / \partial r_{i \alpha}$.

For the bath DOFs, we find two sets of equations which can be solved analytically, since the Lagrangian $\mathcal{L}_{\text {bath, }(v)}+$ $\mathcal{L}_{\text {int, }(v)}\left(\mathbf{r}, \mathbf{u}_{v}\right)$ is harmonic in the bath DOFs $\mathbf{u}_{v}$. These sets of equations are given by

$$
\mu_{l_{v}} \ddot{u}_{b_{v}}=-\sum_{b_{v}^{\prime}} \sqrt{\mu_{l_{v}} \mu_{l_{v}^{\prime}}} D_{b_{v}, b_{v}^{\prime}}^{(v)} u_{b_{v}^{\prime}}-\mu_{l_{v}} f_{b_{v}}(\mathbf{r})
$$

for $v=1,2$. Equation (6) can be solved by introducing the kernel of the differential equation defined from the eigenstates $v_{b_{v}}^{(\lambda)}$ and eigenvalues $\omega_{v, \lambda}^{2}$ of the dynamical matrix $D_{b_{v}, b_{v}^{\prime}}$. The solution of Eq. (6) is then substituted into Eq. (5) to obtain a closed equation in terms of the system DOFs only.

We consider the initial positions and velocities of the bath atoms, appearing in the solution of Eq. (6), being stochastic. It permits us to derive a generalized Langevin-like equation of motion (EOM) for the system DOFs [42,52]:

$$
\begin{aligned}
m_{i} \ddot{r}_{i \alpha}= & -\frac{\partial \bar{V}(\mathbf{r})}{\partial r_{i \alpha}}-\int_{-\infty}^{t} d t^{\prime} \sum_{\nu, i^{\prime} \alpha^{\prime}} K_{i \alpha, i^{\prime} \alpha^{\prime}}^{(v)}\left(t, t^{\prime} ; \mathbf{r}\right) \dot{r}_{i^{\prime} \alpha^{\prime}}\left(t^{\prime}\right) \\
& +\sum_{\nu} \eta_{i \alpha}^{(v)}(t ; \mathbf{r}) .
\end{aligned}
$$

The dynamics of the system DOFs is governed by an effective potential $\bar{V}$, two memory kernels $K_{i \alpha, i^{\prime} \alpha^{\prime}}^{(v)}\left(t, t^{\prime} ; \mathbf{r}\right)$, and stochastic forces $\eta_{i \alpha}^{(\nu)}(t ; \mathbf{r})$ corresponding to each independent bath $v$.

The potential energy $\bar{V}$ is given by the nominal potential energy $V$ inside the central system plus the potential energy between the central system and the two frozen baths. There is also a "polaronic" correction energy due to the coupling between the system atoms and the harmonic displacements of the baths' atoms around their equilibrium positions:

$$
\bar{V}(\mathbf{r})=V(\mathbf{r})-\frac{1}{2} \sum_{\nu} \sum_{b_{v}, b_{v}^{\prime}} \sqrt{\mu_{l_{v}} \mu_{l_{v}^{\prime}}} f_{b_{v}}(\mathbf{r}) \Pi_{b_{v} b_{v}^{\prime}}(0) f_{b_{v}^{\prime}}(\mathbf{r}) .
$$

The memory kernel for the bath $v$ is given by

$$
\begin{array}{r}
K_{i \alpha, i^{\prime} \alpha^{\prime}}^{(v)}\left(t, t^{\prime} ; \mathbf{r}\right)=\sum_{b_{v}, b_{v}^{\prime}} \sqrt{\mu_{l_{v}} \mu_{l_{v}^{\prime}}} g_{i \alpha, b_{v}}(\mathbf{r}(t)) \\
\Pi_{b_{v}, b_{v}^{\prime}}\left(t-t^{\prime}\right) g_{i^{\prime} \alpha^{\prime}, b_{v}^{\prime}}\left(\mathbf{r}\left(t^{\prime}\right)\right) .
\end{array}
$$

The polarization matrix $\Pi_{b_{v}, b_{v}^{\prime}}\left(t-t^{\prime}\right)$ entering the above definitions is obtained from the eigenstates and eigenvalues of the dynamical matrix of the corresponding bath $v$ as follows:

$$
\Pi_{b_{v}, b_{v}^{\prime}}\left(t-t^{\prime}\right)=\sum_{\lambda} \frac{v_{b_{v}}^{(\lambda)} v_{b_{v}^{\prime}}^{(\lambda)}}{\omega_{v, \lambda}^{2}} \cos \left[\omega_{\nu, \lambda}\left(t-t^{\prime}\right)\right] .
$$

Finally, the stochastic (and hence nonconservative) forces $\eta_{i \alpha}^{(\nu)}(t ; \mathbf{r})$ are functions of the initial positions and velocities of the DOFs of the bath $v$. Following Refs. [42,52], we can now assume that each bath $v$, described by the combined Lagrangian $\mathcal{L}_{\text {bath, }(v)}+\mathcal{L}_{\text {int, }(v)}$, is in thermodynamic equilibrium at temperature $T_{v}$. Therefore, the stochastic forces $\eta_{i \alpha}^{(v)}(t ; \mathbf{r})$ can be treated as random variables. From these assumptions, the dissipative forces are well described by a multidimensional Gaussian stochastic process with correlation functions [42,52]

$$
\begin{gathered}
\left\langle\eta_{i \alpha}^{(v)}(t ; \mathbf{r})\right\rangle=0, \\
\left\langle\eta_{i \alpha}^{(v)}(t ; \mathbf{r}) \eta_{i^{\prime} \alpha^{\prime}}^{\left(v^{\prime}\right)}\left(t^{\prime} ; \mathbf{r}\right)\right\rangle=\delta_{\nu \nu^{\prime}} k_{B} T K_{i \alpha, i^{\prime} \alpha^{\prime}}^{(v)}\left(t, t^{\prime} ; \mathbf{r}\right) .
\end{gathered}
$$

\section{B. Extended Langevin dynamics with auxiliary DOFs}

Following Ref. [52], we transform the GLE given by Eq. (7) into a more convenient set of Markovian Langevin dynamics (with white noise) by introducing a set of auxiliary DOFs for each bath $[36-38,43,65,66]$.

First we proceed with a mapping of the memory kernel of each bath by transforming the $\Pi_{b_{v}, b_{v}^{\prime}}$ matrices as follows [52,53]:

$\Pi_{b_{v}, b_{v}^{\prime}}\left(t-t^{\prime}\right) \rightarrow \sum_{k_{v}=1}^{N_{v}^{\mathrm{aDOF}}} c_{b_{v}}^{\left(k_{v}\right)} c_{b_{v}^{\prime}}^{\left(k_{v}\right)} e^{-\left|t-t^{\prime}\right| / \tau_{k_{v}}} \cos \left(\omega_{k_{v}}\left|t-t^{\prime}\right|\right)$.

We then introduce two sets of auxiliary DOFs (aDOFs) $\left\{s_{v, 1}^{\left(k_{v}\right)}(t), s_{v, 2}^{\left(k_{v}\right)}(t)\right\}$ corresponding to each independent bath $v=1,2$. They are associated with the corresponding mapping coefficients $\left\{\tau_{k_{1}}, \omega_{k_{1}}, c_{b_{1}}^{\left(k_{1}\right)}\right\}$ with $k_{1}=1,2, \ldots, N_{1}^{\mathrm{aDOF}}$ and $\left\{\tau_{k_{2}}, \omega_{k_{2}}, c_{b_{2}}^{\left(k_{2}\right)}\right\}$ with $k_{2}=1,2, \ldots, N_{2}^{\mathrm{aDOF}}$.

Note that the frequencies $\omega_{k_{v}}$ used in the mapping Eq. (13) of the matrix $\Pi_{b_{v}, b_{v}^{\prime}}$ are not directly related to the eigenvalues $\omega_{v, \lambda}^{2}$ of the dynamical matrix $D_{b_{v}, b_{v}^{\prime}}^{(v)}$, as explained in detail in Ref. [53]. There would be a one-to-one correspondence only when the number of aDOFs, $N_{v}^{\mathrm{aDOF}}$, is exactly equal to the number of eigenvalues.

For a memory kernel of the type given in Eq. (13), solving the GLE Eq. (7) is equivalent to solving the following extended variable dynamics [52]:

$$
\begin{aligned}
\dot{r}_{i \alpha}= & p_{i \alpha} / m_{i}, \\
\dot{p}_{i \alpha}= & -\frac{\partial \bar{V}}{\partial r_{i \alpha}}+\sum_{b_{1}, k_{1}} \sqrt{\frac{\mu_{l_{1}}}{\bar{\mu}_{1}}} g_{i \alpha, b_{1}}(\mathbf{r}) c_{b_{1}}^{\left(k_{1}\right)} s_{1,1}^{\left(k_{1}\right)}(t) \\
& +\sum_{b_{2}, k_{2}} \sqrt{\frac{\mu_{l_{2}}}{\bar{\mu}_{2}}} g_{i \alpha, b_{2}}(\mathbf{r}) c_{b_{2}}^{\left(k_{2}\right)} s_{2,1}^{\left(k_{2}\right)}(t), \\
\dot{s}_{v, 1}^{\left(k_{v}\right)}= & -\frac{s_{v, 1}^{\left(k_{v}\right)}}{\tau_{k_{v}}}+\omega_{k_{v}} s_{v, 2}^{\left(k_{v}\right)}-\sum_{i \alpha, b_{v}} \sqrt{\mu_{l_{v}} \bar{\mu}_{v}} g_{i \alpha, b_{v}}(\mathbf{r}) c_{b_{v}}^{\left(k_{v}\right)} \dot{r}_{i \alpha} \\
& +\sqrt{\frac{2 k_{B} T_{v} \bar{\mu}_{v}}{\tau_{k_{v}}}} \xi_{v, 1}^{\left(k_{v}\right)}(t) \text { for } v=1,2, \\
\dot{s}_{v, 2}^{\left(k_{v}\right)}= & -\frac{s_{v, 2}^{\left(k_{v}\right)}}{\tau_{k_{v}}}-\omega_{k_{v}} s_{v, 1}^{\left(k_{v}\right)}+\sqrt{\frac{2 k_{B} T_{v} \bar{\mu}_{v}}{\tau_{k_{v}}}} \xi_{v, 2}^{\left(k_{v}\right)}(t) \text { for } v=1,2 .
\end{aligned}
$$


The corresponding total vector state $\boldsymbol{X}=$ $\boldsymbol{X}\left[r_{i \alpha}, p_{i \alpha}, s_{1,1}^{\left(k_{1}\right)}, s_{1,2}^{\left(k_{1}\right)}, s_{2,1}^{\left(k_{2}\right)}, s_{2,2}^{\left(k_{2}\right)}\right] \quad$ follows a multivariate Markovian process, where $\xi_{v, x}^{\left(k_{v}\right)}$ are independent Wiener stochastic processes with (white noise) correlation functions

$$
\begin{aligned}
\left\langle\xi_{v, x}^{\left(k_{v}\right)}(t)\right\rangle & =0, \\
\left\langle\xi_{v, x}^{\left(k_{v}\right)}(t) \xi_{v^{\prime}, x^{\prime}}^{\left(k_{v^{\prime}}^{\prime}\right)}\left(t^{\prime}\right)\right\rangle & =\delta_{v v^{\prime}} \delta_{x x^{\prime}} \delta_{k_{v} k_{v}^{\prime}} \delta\left(t-t^{\prime}\right) .
\end{aligned}
$$

We recall that even if the total vector state $\boldsymbol{X}$ corresponds to Markovian processes, a subset of its components, for example the vector $\overline{\boldsymbol{X}}=\overline{\boldsymbol{X}}\left[r_{i \alpha}, p_{i \alpha}\right]$, is not necessarily following Markovian processes [61]. This was clearly shown in the previous section where the random noise of the corresponding GLE is actually a colored noise given by the memory kernel of the GLE. For such classes of non-Markovian processes (which are components, or functions of one or more components, of multivariate Markovian processes) self-consistency is guaranteed as the Chapman-Kolmogorov equation is satisfied [61].

\section{Integration algorithm from a Fokker-Planck approach}

Following the scheme given in Ref. [52], we now develop a Fokker-Planck (FP) approach to derive a set of equations which are equivalent to the equations of the extended Langevin dynamics given by Eq. (14). We consider the probability distribution function (PDF) $P\left(r_{i \alpha}, p_{i \alpha},\left\{s_{v, 1}^{\left(k_{v}\right)}, s_{v, 2}^{\left(k_{v}\right)}\right\}, t\right)$ of the total vector state $\boldsymbol{X}$. Such a PDF follows a FP dynamical equation which can be written as follows:

$\dot{P}\left(r_{i \alpha}, p_{i \alpha},\left\{s_{v, 1}^{\left(k_{v}\right)}, s_{v, 2}^{\left(k_{v}\right)}\right\}, t\right)=-\hat{\mathfrak{L}}_{\mathrm{FP}} P\left(r_{i \alpha}, p_{i \alpha},\left\{s_{v, 1}^{\left(k_{v}\right)}, s_{v, 2}^{\left(k_{\nu}\right)}\right\}, t\right)$,

where $\hat{\mathfrak{L}}_{\mathrm{FP}}$ is the corresponding FP Liouvillian.

We split the Liouvillian $\hat{\mathfrak{L}}_{\mathrm{FP}}$ in two parts: a conservative part $\hat{\mathfrak{L}}_{\text {cons }}$ and a dissipative part $\hat{\mathfrak{L}}_{\text {diss }}$. The dynamics generated by the conservative part $\hat{\mathfrak{L}}_{\text {cons }}$ corresponds to the EOM of the DOFs and aDOFs given in Eq. (14) if one omits all the terms containing the $\tau_{k_{v}}$ parameters [52]. The remaining dissipative part $\hat{\mathfrak{L}}_{\text {diss }}$ generates the EOM of the aDOFs, given by the following generic form:

$$
\dot{s}_{v, x}^{\left(k_{v}\right)}(t)=-\frac{s_{v, x}^{\left(k_{v}\right)}(t)}{\tau_{k_{v}}}+\sqrt{\frac{2 k_{B} T_{v} \bar{\mu}_{v}}{\tau_{k_{v}}}} \xi_{v, x}^{\left(k_{v}\right)}(t) .
$$

For such a stochastic dynamics there exists an exact integration algorithm [52,62].

In order to obtain an integration algorithm (see details in Appendix A), we consider the time evolution of the PDF over an elementary time step $\Delta t$ :

$$
P(\{\ldots\}, t+\Delta t)=e^{-\hat{\mathfrak{L}}_{\mathrm{FP}} \Delta t} P(\{\ldots\}, t) .
$$

We use the splitting of $\hat{\mathfrak{L}}_{\mathrm{FP}}$ and a second-order Trotter expansion to decompose the time evolution operator as follows [63]:

$$
e^{-\hat{\mathfrak{L}}_{\mathrm{FP}} \Delta t} \sim e^{-\hat{\mathfrak{L}}_{\mathrm{diss}} \frac{\Delta t}{2}} e^{-\hat{\mathfrak{L}}_{\mathrm{cons}} \Delta t} e^{-\hat{\mathfrak{L}}_{\mathrm{diss}} \frac{\Delta t}{2}} .
$$

The first and last time evolution operators $e^{-\hat{\mathfrak{L}}_{\text {diss }} \frac{\Delta t}{2}}$ with half a time step $\Delta t / 2$ generate steps $(\mathrm{A})$ and $(\mathrm{H})$ in the algorithm given in Appendix A.

Furthermore, we use a second Trotter expansion of the term $e^{-\hat{\mathfrak{L}}_{\text {cons }} \Delta t}$ by splitting $\hat{\mathfrak{L}}_{\text {cons }}$ in two parts $-\hat{\mathfrak{L}}_{r, s_{1}}$ and $\hat{\mathfrak{L}}_{p, s_{2}}$. The part in $\hat{\mathfrak{L}}_{p, s_{2}}$ generates the time evolution of the system DOFs $p_{i \alpha}$ and of the aDOFs $s_{v, 2}^{\left(k_{v}\right)}$ over half a time step $\Delta t / 2$; see steps (C) and step (G) in Appendix A. The part in $\hat{\mathfrak{L}}_{r, s_{1}}$ generates the time evolution of the system DOFs $r_{i \alpha}$ and of the aDOFs $s_{v, 1}^{\left(k_{v}\right)}$ over $\Delta t$; see steps (D) and (F) in Appendix A.

\section{Calculation of the polarization matrices $\Pi_{b_{v} b_{v}^{\prime}}$}

In order to perform the mapping given by Eq. (13), we first Fourier-transform the equation into

$$
\begin{aligned}
\Pi_{b_{v}, b_{v}^{\prime}}(\omega)= & \sum_{k_{v}} c_{b_{v}}^{\left(k_{v}\right)} c_{b_{v}^{\prime}}^{\left(k_{v}\right)} \\
& \times\left[\frac{\tau_{k_{v}}}{1+\left(\omega-\omega_{k_{v}}\right)^{2} \tau_{k_{v}}^{2}}+\frac{\tau_{k_{v}}}{1+\left(\omega+\omega_{k_{v}}\right)^{2} \tau_{k_{v}}^{2}}\right] .
\end{aligned}
$$

For each bath, the set of parameters $\left\{c_{b_{v}}^{\left(k_{v}\right)}, \tau_{k_{v}}, \omega_{k_{v}}\right\}$ is obtained from a fitting procedure (described in detail in Ref. [53]) based on the vibrational properties of the bath. As shown in Ref. [53], the polarization matrices $\Pi_{b_{v} b_{v}^{\prime}}(\omega)$ are related to the imaginary part of the phonon bath propagator $\mathcal{D}_{b_{v} b_{v}^{\prime}}(\omega)$ as follows:

$$
\Pi_{b, b^{\prime}}(\omega)=-2 \operatorname{Im}_{\mathcal{D}_{b, b^{\prime}}}(\omega) /|\omega| .
$$

The bath propagator is defined from the dynamical matrix $\boldsymbol{D}^{(v)}$ of the bath $v$ as

$$
\mathcal{D}_{b_{v}, b_{v}^{\prime}}(\omega)=\left[\omega^{2} \mathbf{1}-\boldsymbol{D}^{(v)}+i \varepsilon\right]_{b_{v}, b_{v}^{\prime}}^{-1},
$$

where $\varepsilon \rightarrow 0^{+}$. We use a real-space method, based on the Lanczos algorithm, to calculate the inverse of the matrix defining $\mathcal{D}_{b_{v}, b_{v}^{\prime}}$ and the fitting procedure, described in Ref. [53], to get the values of the parameters $\left\{c_{b_{v}}^{\left(k_{v}\right)}, \tau_{k_{v}}, \omega_{k_{v}}\right\}$.

Once the system is defined, the calculations of the dynamical matrices and the mapping procedures are performed individually for each bath $v$.

\section{E. Generalization to $N_{\text {bath }}$ independent baths}

It should be noted that the generalization to the case of the central system connected to $N_{\text {bath }}$ independent baths is straightforward. One can expand the GLE-2B by introducing $N_{\text {bath }}$ auxiliary sets of DOFs $s_{v, 1}^{\left(k_{v}\right)}$ and $s_{v, 2}^{\left(k_{v}\right)}$. The EOMs of these $\mathrm{aDOFs}$ are given in the third and fourth lines of Eq. (14). The EOM for the momenta of the central system will include the contribution of the $N_{\text {bath }}$ baths via the set of aDOFs. It is defined by the sum over all the bath indices instead of just a sum over $v=1,2$, i.e.,

$$
\dot{p}_{i \alpha}=-\frac{\partial \bar{V}}{\partial r_{i \alpha}}+\sum_{\nu=1}^{N_{\text {bath }}} \sum_{b_{v}, k_{v}} \sqrt{\frac{\mu_{l_{v}}}{\bar{\mu}_{\nu}}} g_{i \alpha, b_{v}}(\mathbf{r}) c_{b_{v}}^{\left(k_{\nu}\right)} s_{\nu, 1}^{\left(k_{\nu}\right)}(t) .
$$

The mapping and fitting procedures of the polarizations matrices $\Pi_{b_{v} b_{v}^{\prime}}$ will be performed for each individual bath.

\section{RESULTS FOR THE GLE-2B APPROACH}

We now apply the GLE-2B approach to a model system, which consists of a short Al nanowire connected to two independent baths (represented by two half spheres) as shown 


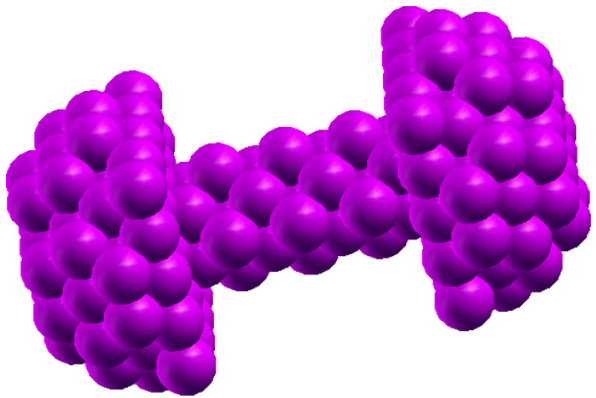

FIG. 2. Model system for a short cylindric Al wire (central system containing 59 atoms) connected to two left $(L)$ and right $(R)$ baths. For the GLE calculations, the $L$ and $R$ bath reduced region contains 68 atoms each. For the calculations of the dynamical matrix and the mapping of the polarization matrix, larger baths (each containing 203 atoms) were considered [53]. The embedded atom method (EAM) is used for the interatomic effective potential. For convenience, we now call the baths $v=L, R$ instead of $v=1,2$.

in Fig. 2. For obvious reasons, we now call the left and right baths $v=L, R$ (instead of $v=1,2$ ). The electronic transport properties of similar Al nanowires were studied some decades ago [67-69]; it is now interesting to study their thermal transport properties under the proper nonequilibrium conditions.

Figure 3 provides more information about the central system (labeling of different layers of the system) and the bath

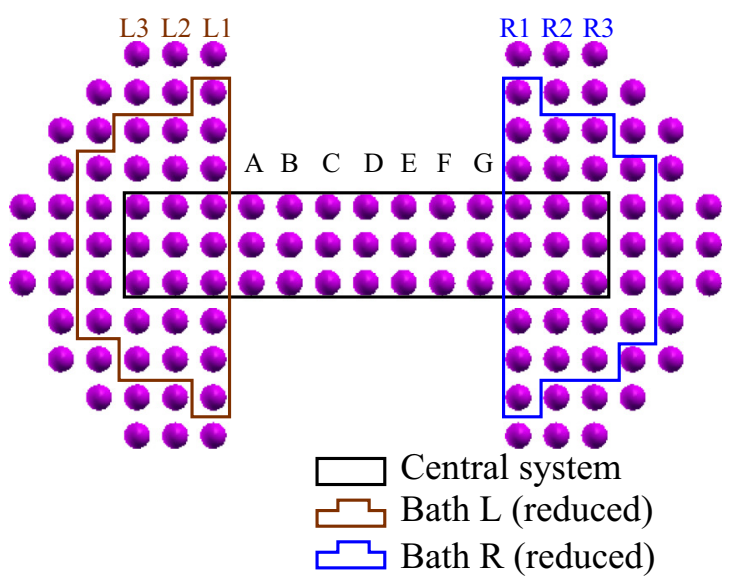

FIG. 3. Schematic representation of systems under consideration for the GLE calculations. The central system consists of 7 layers of $\mathrm{Al}$ (labeled A to G) and 3 extra layers embedded in the left bath (labeled L1, L2, and L3) and 3 extra layers embedded in the right bath (labeled R1, R2, and R3). The layers L3, L1, B, D, F, R1, and R3 (L2, A, C, E, G, R2) each contain 5 (4) Al atoms. The bath reduced regions are represented by the brown and blue regions for the left and right baths, respectively. Each of these regions contains 68 atoms. We recall that, during the GLE simulations, the positions of the bath atoms are fixed. The left and right baths are at their own temperature $T_{L}$ and $T_{R}$, respectively. For the other thermostatting approaches, the bath reduced regions are described by their own stochastic dynamics [Langevin-Gauss (LG) or Nosé-Hoover (NH) thermostatting] at their own temperatures $T_{L}$ and $T_{R}$. The central system (L1-L3, A-G, R1-R3) evolves according to a Newtonian (NVE) dynamics. The remaining outer atoms are kept at fixed positions to ensure the structural stability of the system in the LG and NH thermostatting calculations. reduced regions. The system is built using a fcc lattice and the distance between layer $\mathrm{A}$ and layer $\mathrm{C}$ (Fig. 3) corresponds to the lattice parameter of $4.05 \AA$. The $\mathrm{Al}$ nanowire (layers A to G) has the length of $12.15 \AA$. The central system (layers L3-L1, A-G, R1-R3) for which the GLE-2B simulations are performed has a length of $20.25 \AA$. We have taken the embedded atom method [70] to model the metallic Al system. The tabulated interatomic potential is provided by the NIST Interatomic Potential Repository Project [71].

In order to compare to the results obtained with our GLE2B approach, we also consider two different thermostatting approaches for the baths. These are more widely used and consist of stochastic dynamics for the atoms in the bath regions, while the central region follows the common deterministic Newtonian dynamics (see Fig. 3 and Appendix A). We consider two stochastic dynamics, i.e., a simple Langevin (Langevin-Gauss, LG) dynamics [58] and the dynamics generated by a Nosé-Hoover $(\mathrm{NH})$ thermostat which are already implemented in the MD code LAMMPS.

For the simple LG approach, the stochastic dynamics for the atoms in the bath regions is given by

$$
\dot{\mathbf{p}}_{v}=-\nabla_{\mathbf{r}_{v}} V(\mathbf{r})-\gamma \mathbf{p}_{v}+\boldsymbol{\xi}_{v}^{G}
$$

with the momentum vector $\mathbf{p}_{v}$ of the atoms in the bath $v=$ $L, R$ and the white noise vector $\xi_{v}^{G}$ (following a Gaussian distribution with a width related to the temperature $T_{v}$ ). The single parameter $\gamma$ characterizes the friction (damping $\tau_{\text {damp }}=$ $1 / \gamma)$ for all atoms in the bath regions [53].

For the NH thermostatting approach, each is also characterized by a damping parameter $\tau_{\mathrm{damp}}^{\mathrm{NH}}$ (in units of time) in the LAMMPS implementation.

As a first application of our GLE-2B, we concentrate on studying the steady-state properties of the system. For all stochastic dynamics, we consider some initial conditions (values of the baths' temperatures $T_{L}$ and $T_{R}$ and of the velocities of the atoms in the central system) and let the system evolve in time until the total kinetic energy reaches a plateau, i.e., a constant value up to the corresponding thermal fluctuations. For the different GLE-2B calculations, the system takes roughly 80 ps to reach a steady-state regime (i.e., $\sim 40000$ time steps for a $\Delta t=2 \mathrm{fs}$ ). For the $\mathrm{LG}$ and $\mathrm{NH}$ thermostat calculations, the steady state can be reached in fewer time steps since the characteristic damping time is adjustable by the user.

\section{A. Systems at equilibrium}

In order to validate our GLE-2B approach and the corresponding algorithm, we first need to check that when the bath temperatures are equal, $T_{L}=T_{R}$, we obtain the correct results for the energy and/or the velocity distributions as expected from equilibrium statistical mechanics.

We have performed different calculations for different temperatures $T=T_{L}=T_{R}$ where $T=100,150,200,300$, $400 \mathrm{~K}$. Figure 4 shows the velocity distribution functions for different atomic layers of the central system [73]. The results for the velocity distributions show that the system indeed reaches the expected thermal equilibrium, where the velocity distributions follow the Maxwell equilibrium distribution.

It should be noted that for temperatures above $\sim 400 \mathrm{~K}$, our model system shows some structural instabilities [74]. 

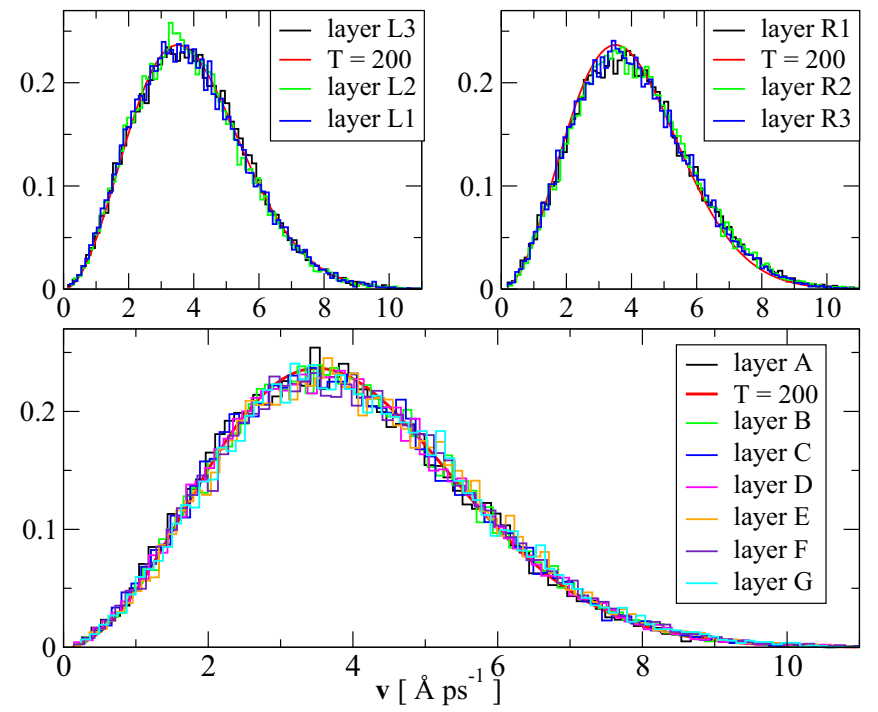

FIG. 4. Histograms of the velocity distribution for different layers of atoms for the system shown in Fig. 3. Top left panel: Layers L3, L2, L1 of the central system. Top right panel: Layers R1, R2, R3 of the central system. Bottom panel: Layers A to G. The GLE calculations are performed for $T_{L}=T_{R}=200 \mathrm{~K}$. All distributions (histograms) fit perfectly the equilibrium Maxwell distribution (solid red line) as expected.

Therefore calculations are performed only with temperatures lower than $400 \mathrm{~K}$.

To further complement the validity of our approach, we have also performed calculations for pseudo-double-equilibrium conditions. This is done by considering the two baths at two different temperatures, and keeping fixed the atomic coordinates of the central layer D of the central system. This creates a thermal decoupling between the two sides; i.e., the frozen layer D acts as a perfect reflective barrier for the thermal transport between the two baths. Figure 5 shows the results obtained for the velocity distribution when the bath temperatures are $T_{L}=200 \mathrm{~K}$ and $T_{R}=125 \mathrm{~K}$ [76]. One clearly sees that one side of the system (layers L3, L2, L1, A, $\mathrm{B}, \mathrm{C})$ has velocity distributions that are well represented by the equilibrium Maxwell distributions obtained for $T_{L}$, while the other side (layers E, F, G, R1, R2, R3) has equilibrium velocity distributions obtained from $T_{R}$. Similar results are also obtained when using the LG or $\mathrm{NH}$ thermostatting procedures for the bath stochastic dynamics.

With this preliminary set of calculations, we feel confident that our approach and its numerical implementation in the MD code LAMMPS are correct and, therefore, we move on to discussing our out-of-equilibrium calculations.

\section{B. Nonequilibrium conditions}

In this section, we consider the proper nonequilibrium conditions when the temperatures of the two baths are different $T_{L} \neq T_{R}$. More specifically, we consider the steady-state regime when, after some relaxation time, the total kinetic energy of the system reaches a "constant" value (up to the thermal fluctuations).

In a nanowire connecting two thermal baths when $T_{L} \neq T_{R}$, it has been shown that a temperature gradient may or may not
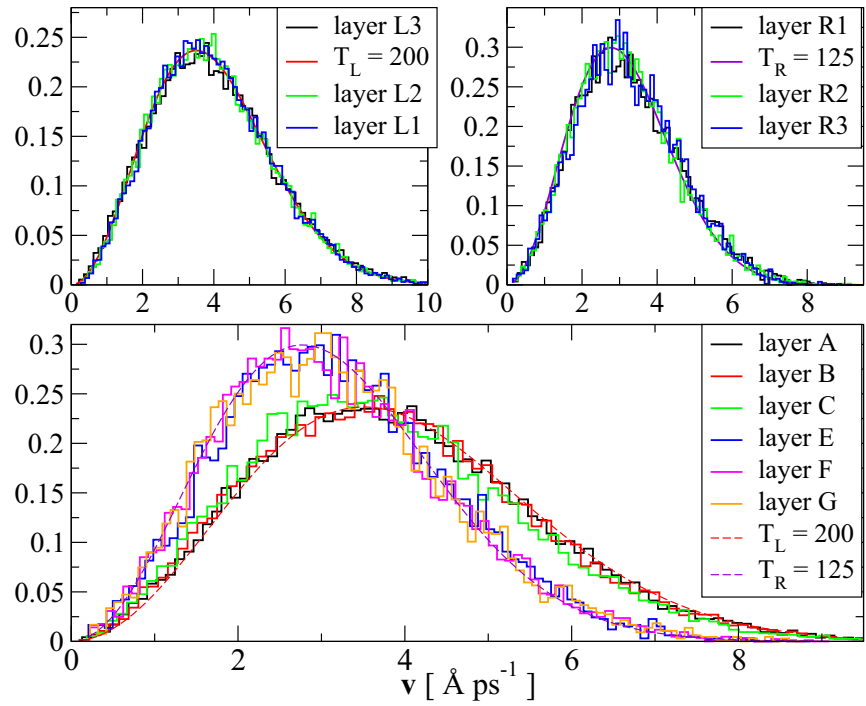

FIG. 5. Histograms of the velocity distribution for the different layers of atoms in the system shown in Fig. 3. The GLE calculations are performed for two different bath temperatures $T_{L}=200 \mathrm{~K}$ and $T_{R}=125 \mathrm{~K}$ [76]. The central layer $\mathrm{D}$ is frozen, i.e., atomic positions fixed, and acts as a perfect thermal barrier. The distributions (histograms) for the atomic layers on both sides of the frozen layer D, namely layers (L3, L2, L1, A, B, C) and layers (E, F, G, R1, R2, R3), fit perfectly the equilibrium Maxwell distributions obtained for the two temperatures $T_{L}$ (lines in the top left and bottom panels) and $T_{R}$ (lines in the top right and bottom panels).

build up across the system. Temperature profile measurements are usually performed, by using thermal probe AFM, on mesoscopic scale systems (a few microns in length) or on multiwall carbon nanotubes (14 nm diameter and 4 microns length) [75]. Such measurements have not yet been performed on nanoscale objects. However, a lot of theoretical work on model systems can be found in the literature. In the following paragraphs, we will review briefly the causes of the presence or absence of a temperature gradient as reported in previous theoretical studies.

Since the seminal work of Rieder et al. [77], it has been known that in a one-dimensional homogeneous harmonic system (also referred to as an integrable system), the thermal conductivity diverges in the thermodynamic limit. No temperature gradient is formed in the bulk of the system, since the dominating energy "carriers" are not scattered and propagate ballistically. A large variety of harmonic (integrable) systems have been studied in the classical [77-85] and quantum [79,8691] limits, using analytical and/or numerical approaches. All these studies show that there is no temperature gradient inside the system (except small regions in the vicinity of the contacts between the central system and the baths). One usually obtains a constant-temperature profile [77-81,83,85-91] in the central system around the averaged temperature $T_{\mathrm{av}}=\left(T_{L}+T_{R}\right) / 2$.

On the other hand, in classical or quantum nonintegrable systems, a temperature gradient is formed inside the system. The temperature gradient is uniform, and the heat conductivity is finite (it means that these systems obey Fourier's law). The general trend is that one has to break the integrability of the system in order to build up a temperature gradient 
there. This can be done by introducing (a) any form of anharmonicity $[79,81,82,84,85,90,92,94,95,97-106]$, (b) extra local stochastic processes $[78,79,83,85,86,94,100,109]$ or extra collision processes [110,111], (c) dephasing for quantum systems [87,112], or (d) mode coupling for classical systems [113].

The introduction of topological/configurational defects $[92,93,107]$ or disorder $[78,83,93,99,108]$ in harmonic systems can also lead to the buildup of a temperature gradient. This result can be understood from the fact that defect/disorder introduces some form of localization of the vibration modes $[95,96]$. Such modes do not favor ballistic transport, as phonons get scattered by impurities or boundaries [95]. Hence the system has a finite thermal conductivity and presents a temperature gradient across itself.

We now test our GLE-2B method to investigate the thermal transport properties of the $\mathrm{Al}$ nanowire in the context of ballistic versus diffusive transport regimes. We consider different temperatures $T_{L}$ and $T_{R}$ for the left and right baths, respectively. The temperatures are chosen between 50 and $300 \mathrm{~K}$.

Figure 6 shows the velocity distribution for a nonequilibrium calculation performed with $T_{L}=150 \mathrm{~K}$ and $T_{R}=50 \mathrm{~K}$. This is a typical result, and the following discussion can be applied to other combinations of bath temperatures $T_{L}$ and $T_{R}$ (not shown in the paper). The corresponding time evolution of the total kinetic energy of the central system and its statistical distribution is shown in Appendix A.

First of all, we can see that the line shape of the different velocity histograms for different atomic layers is similar to the line shape of the equilibrium Maxwell distribution. Such a behavior permits us to define a local temperature for each different layer.

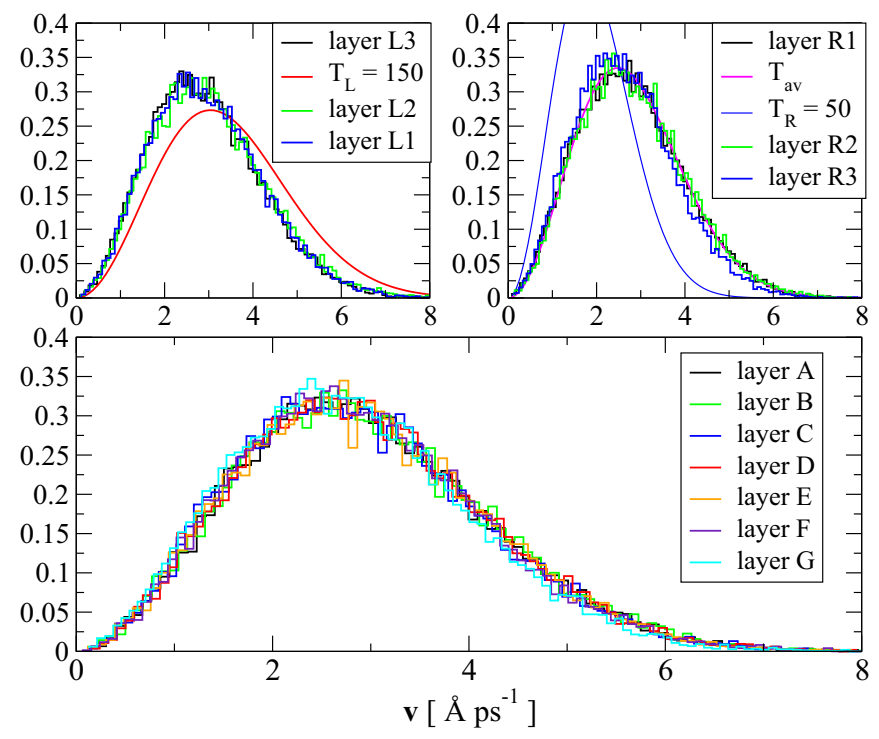

FIG. 6. Histograms of the velocity distribution for different layers of atoms for the system shown in Fig. 3. The GLE calculations are performed for proper nonequilibrium conditions with the bath temperatures being $T_{L}=150 \mathrm{~K}$ and $T_{R}=50 \mathrm{~K}$. The line shape of the different distributions (histograms) is similar to that of the equilibrium Maxwell-Boltzmann distribution. All distributions fit well onto the Maxwell distribution obtained from the average temperature $T_{\mathrm{av}}=\left(T_{L}+T_{R}\right) / 2=100 \mathrm{~K}$.
Second, all velocity histograms appear to follow the same equilibrium Maxwell distribution corresponding to the average temperature $T_{\mathrm{av}}=\left(T_{L}+T_{R}\right) / 2=100 \mathrm{~K}$ (even for the layers L3 and R3 embedded in the $L$ and $R$ bath, respectively). The overall results clearly indicate that, within such a nonequilibrium regime, we are dealing with an integrable system, essentially a complex harmonic system which perfectly transmits the heat flux between the two baths.

To simplify the analysis of our calculations, we now consider only the temperature profiles along the central system. Such profiles are calculated for each layer by fitting the local velocity histograms onto the Maxwell distribution. We then extract the corresponding local temperature for each layer.

Figure 7 shows the temperature profiles in the central system for different sets of temperatures $T_{L, R}$. From the procedure of fitting the velocity histograms to the Maxwell distribution, we estimate an uncertainty of approximately $\pm 5 \mathrm{~K}$ on the temperature values. Even in the presence of such an error, one can see that there is no temperature gradient in the central system for the different temperatures considered in Fig. 7. This implies that, for such temperatures, the system behaves like a perfect harmonic (integrable) thermal conductor.

We can also compare our GLE-2B approach with the other LG and NH thermostatting approaches (Fig. 11). In such approaches, the atoms in the bath reduced regions are allowed to move and follow a dissipative dynamics ruled by a simple LG dynamics or by a NH thermostat. The atoms in the central region (while interacting with themselves and with the moving atoms of the two baths) follow a Newtonian NVE dynamics.

From the results shown in Fig. 8, we can see that while the GLE-2B calculations provide a uniform temperature profile inside the system, the LG and NH thermostatting approaches display two different kinds of behavior depending on the

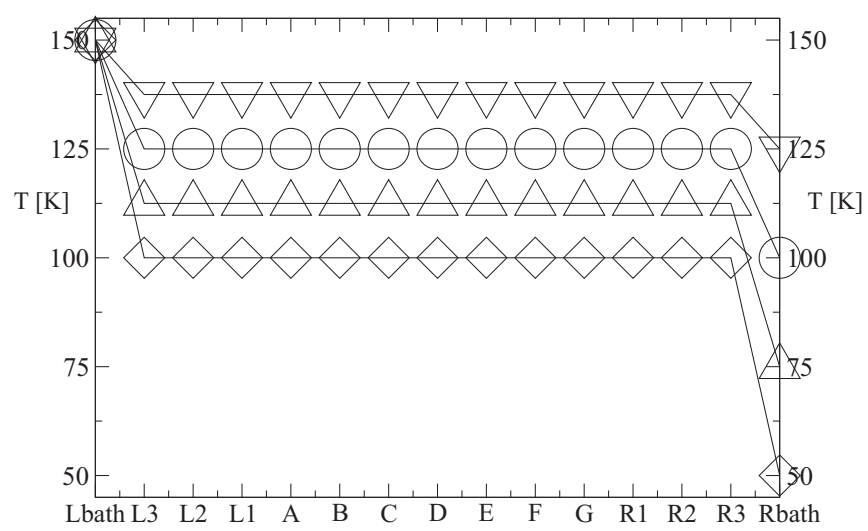

FIG. 7. Temperature profiles across the central system for different bath temperatures. The labels Lbath (Rbath) represent the temperatures at the left (right) bath. The other labels indicate different layers in the central system. For different sets of $T_{L, R}$, there is no temperature gradient in the system; i.e., it behaves like a perfectly integrable system. There is an inherent uncertainty in the evaluation of the local temperature from a fit to the Maxwell distribution. All temperatures have an error bar of $\pm 5 \mathrm{~K}$. As a visual guide, this corresponds to the size of the symbols. Note that we also have performed calculations swapping $T_{L} \leftrightarrow T_{R}$ and obtained the same flat temperature profiles. 
chosen value of damping parameter. Note that in all LG and NH calculations, we have performed the calculations with as many time steps as required to reach a stationary state. The larger the value of the damping parameter, the longer the dynamics is needed to reach the stationary state (see also Appendix A for the time evolution of the kinetic energy). For small values of the damping parameter $\tau_{\text {damp }}=7$ ps $\left(\tau_{\text {damp }}^{\mathrm{NH}}=0.7 \mathrm{ps}\right)$, we obtain a temperature gradient in the central system. The $\mathrm{NH}$ thermostats appear to provide an almost perfect linear gradient in the whole system, while the simple Langevin thermostatting corresponds to a small temperature gradient. However for larger values of the damping parameter $\tau_{\text {damp }}=$ $100 \mathrm{ps}\left(\tau_{\mathrm{damp}}^{\mathrm{NH}}=20 \mathrm{ps}\right)$, both LG and NH thermostats provide a flatter temperature profile. The LG calculations result in a temperature profile almost similar to that of the GLE-2B calculations. It is interesting to see that changing the value of the damping can lead to completely different physical results, i.e., the presence of a temperature gradient or its absence in the central system. Such a dilemma does not exist in our GLE-2B approach since it does not contain any adjustable parameter. One should note that the small values of the damping parameter have been chosen in order to reproduce a relaxation of the kinetic energy (for the LG and NH thermostatting) similar to the relaxation obtained from the GLE-2B approach (see also Appendix A). It seems fair to say that fitting the values of the damping parameter (to reproduce the evolution of the kinetic energy) is not enough for obtaining the same temperature profiles with the LG and NH thermostats and with the GLE-2B approach. As mentioned above, the presence of a temperature gradient is a signature of the breaking down of the integrability

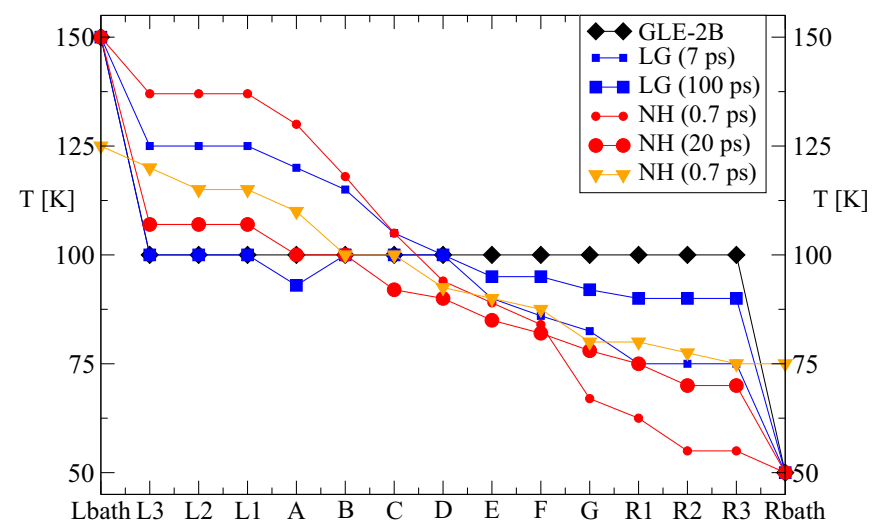

FIG. 8. Temperature profiles across the system for different bath temperatures $T_{L}=150, T_{R}=50$. Comparison between the GLE-2B approach and the other $\mathrm{LG}$ and $\mathrm{NH}$ thermostatting approaches. While the GLE-2B calculations provide a uniform temperature profile inside the system, the LG and NH thermostats show either the building up of a temperature gradient in the central system or a flatter temperature profile depending on the value used for the damping parameter. The LG calculations performed with the damping parameter $\tau_{\mathrm{damp}}=1 / \gamma=7$ ps show a temperature gradient, while almost no gradient is obtained for $\tau_{\text {damp }}=100$ ps. The $\mathrm{NH}$ calculations show a strong temperature gradient for $\tau_{\mathrm{damp}}^{\mathrm{NH}}=0.7 \mathrm{ps}$, and a flatter temperature gradient for $\tau_{\mathrm{damp}}^{\mathrm{NH}}=20 \mathrm{ps}$. There is still a gradient for $\mathrm{NH}$ thermostats (with $\tau_{\mathrm{damp}}^{\mathrm{NH}}=0.7 \mathrm{ps}$ ) even for smaller $\Delta T$ with $T_{L}=125$ and $T_{R}=75$ (see orange curve). of the system, and corresponds to a system with a finite thermal conductance. It also implies the introduction of anharmonic effects, configurational disorder, or the introduction of other (uncontrolled) random processes.

In order to understand whether one can obtain a temperature gradient with the GLE-2B approach, we have performed calculations in different situations where the harmonicity of the system is broken. This can be achieved by considering higher temperatures. In these cases, the atoms of the central system move in an effective potential which goes beyond the harmonic potential well and consequently one can obtain a finite temperature gradient, as shown in Fig. 9. One can see that larger deviations of the temperatures from the average $T_{\mathrm{av}}=\left(T_{L}+T_{R}\right) / 2$ are obtained on the side of the hotter bath. One can further increase such effects by introducing artificially some configurational disorder in the system. For that, we have considered the same system and at random we have given one atom in each layer L1, A-G, and R1 a mass $20 \%$ larger (smaller) than the mass of the other atoms in the system. The corresponding temperature profile is shown in Fig. 9 and presents, as expected for such a disordered and/or anharmonic system, a finite temperature gradient. In Appendix D we present calculations for a model of a one-dimensional Al chain. The GLE-2B calculations obtained for such a simple model confirm, as expected, the analysis we have performed in this section.

Furthermore, it is also crucial to understand the importance of anharmonic effects. For that we introduce into the GLE-2B method some form of anharmonic effects in the baths by taking a finite lifetime for the phonon modes of the bath. It means that vibrational excitations do not have an infinite lifetime (as should be the case for an integrable harmonic system) but rather that they decay (dissipate) in time. The simplest way to treat such effects is to introduce a "self-energy" in the phonon

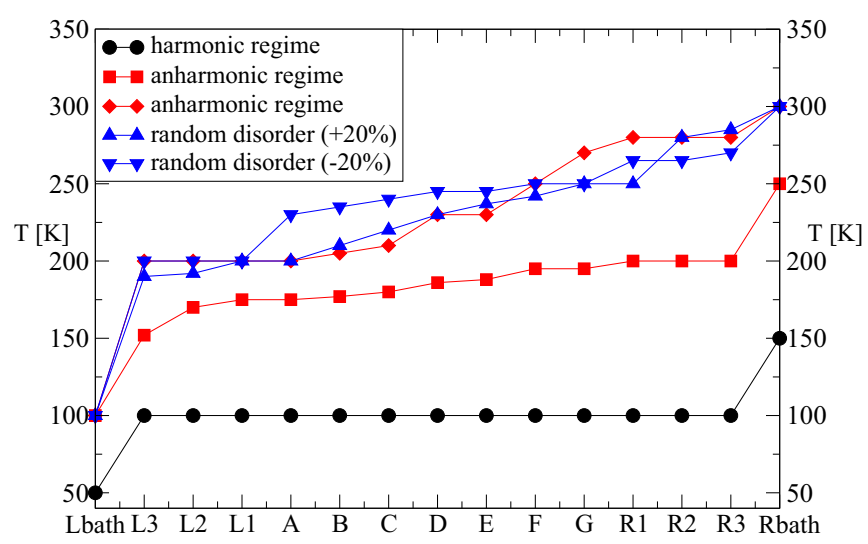

FIG. 9. Temperature profiles across the system from the GLE2B calculations under different $T_{L, R}$ and different atomic masses. It is possible to break the integrability of the system by considering larger temperatures (red curves) and/or by introducing configurational disorder (blue curve). The disorder is introduced by changing the mass $( \pm 20 \%)$ of some atoms picked up randomly in the central system. For higher temperatures, parts of the system are "driven" beyond the harmonic limit. In such cases, the system is not entirely ballistic and presents a finite thermal conductivity, leading to the buildup of a temperature gradient. 


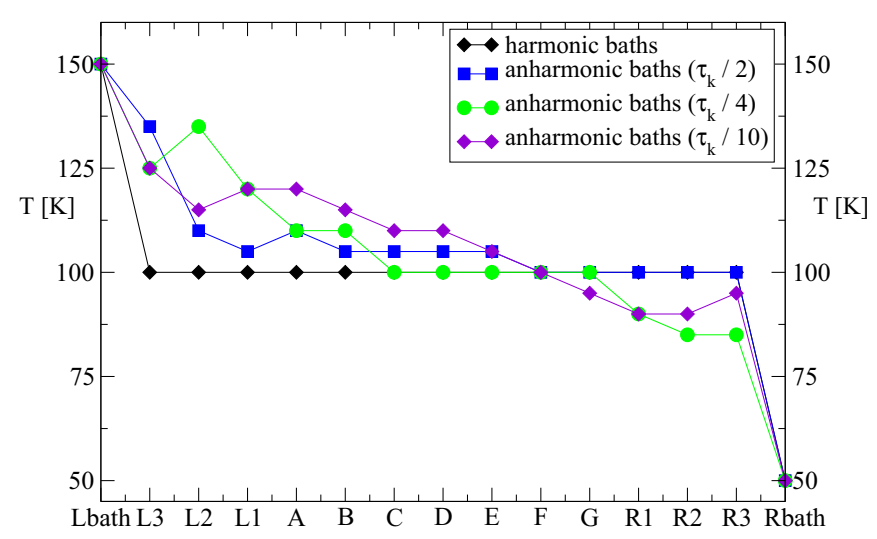

FIG. 10. Temperature profiles across the system from the GLE2B calculations. A simplified form of anharmonic effects in the baths is introduced by modifying the values of the fitted parameters $\tau_{k_{\nu}}$. Such anharmonic effects lead to a small temperature gradient on the side of the hotter bath ( $L$ side). The building up of the temperature gradient is however not as strong as for the LG/NH thermostatting calculations.

bath propagator,

$$
\mathcal{D}_{b_{v}, b_{v}^{\prime}}(\omega)=\left[\omega^{2} \mathbf{1}-\boldsymbol{D}^{(v)}+\omega \boldsymbol{\Sigma}^{\mathrm{anh}}(\omega)\right]_{b_{v}, b_{v}^{\prime}}^{-1} .
$$

Furthermore, we consider that $\boldsymbol{\Sigma}^{\text {anh }}$ is purely imaginary and simply modifies the linewidth of the spectral features in $\Pi_{b, b^{\prime}}(\omega)=-2 \operatorname{Im} \mathcal{D}_{b, b^{\prime}}(\omega) /|\omega|$. In practice, such effects can be implemented in a rather straightforward way: once the mapping of $\Pi_{b, b^{\prime}}(\omega)$ is established, we take the values of the fitted parameters $\tau_{k_{v}}$ and make them smaller. The features of the corresponding vibration modes in the phonon bath propagator are then broadened [53].

The results of such calculations, shown in Fig. 10, suggest that anharmonic effects in the baths tend to favor the buildup of the temperature gradient across the system.

Finally we would like to add that short nanowires can be ballistic (harmonic regime) for a range of temperatures $T_{L, R}$. However, such a behavior might not be true for much larger (longer) and strongly heterogeneous systems [22]. Indeed, in such systems, one may expect to observe the presence of disorder, of more localized vibrational modes, or, more importantly, of vibrational mode "mixing" effects (interaction between phonons) which lead to the building up of a temperature gradient in the system. For example, the process of mode coupling has been studied in model three-dimensional systems [113] and has been shown to lead to the presence of a temperature gradient in the system.

\section{CONCLUSION}

We have developed a generalized Langevin equation (GLE) approach to treat nonequilibrium conditions when a central classical region is connected to two realistic thermal baths at two different temperatures. The method is called GLE-2B for generalized Langevin equation with two baths. Following the original GLE approach [52,53], the extended Langevin dynamics scheme is modified to take into account two sets of auxiliary degrees of freedom, each of which characterizes the vibrational properties of the baths. These auxiliary degrees of
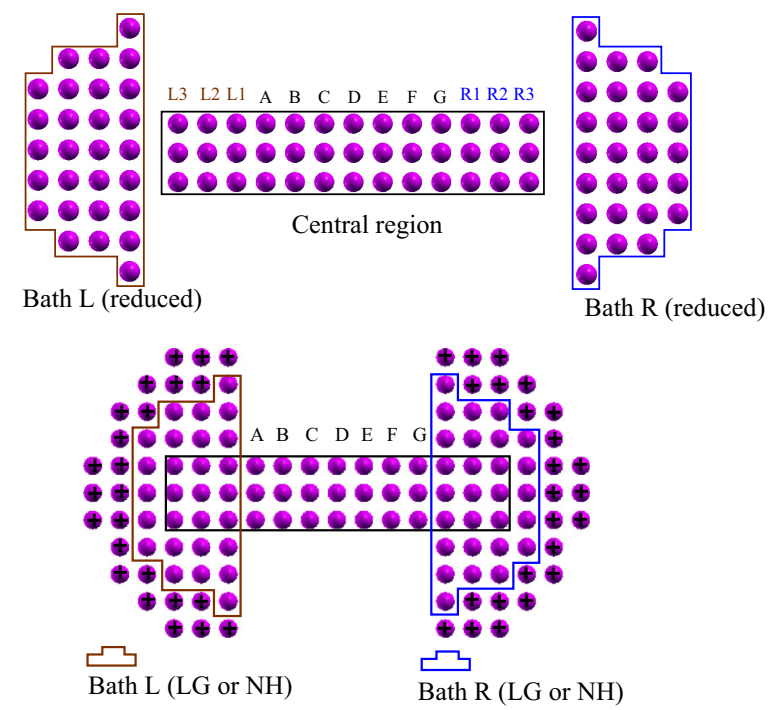

FIG. 11. Schematic description of the GLE-2B and the LG/NH calculations. Upper panel: The GLE dissipative dynamics Eq. (7) is performed only on the atoms of the central region. The $L$ and $R$ baths (displaced farther to the left and right for clarity) enter into the GLE calculations via the sets of fitting parameters $\left\{c_{b_{\nu}}^{\left(k_{\nu}\right)}, \tau_{k_{\nu}}, \omega_{k_{\nu}}\right\}$ and the $g_{i \alpha, b_{v}}\left(\left\{r_{i \alpha}\right\}\right)$ quantities. In such calculations, the positions of the bath atoms are fixed at their equilibrium positions shown in Fig. 3. Lower panel: The atoms in the central region follow Newton's EOM and the atoms in the $L$ and $R$ baths follow a dissipative $\mathrm{LG}$ or NH dynamics. The atoms with a black cross are kept fixed to ensure the overall stability of the system. The two approaches, GLE-2B and LG/NH, represent two different kinds of stochastic processes.

freedom are then used to solve the non-Markovian dissipative dynamics of the central region. We have developed the corresponding algorithm for MD simulations and implemented it within the MD code LAMMPS.

As a first application, we have studied the heat transport properties of a short Al nanowire, that connects the left and the right $\mathrm{Al}$ baths, in the steady-state regime. We have mostly considered the establishment of a local temperature profile within the system when the two bath temperatures are different. Our results are interpreted in terms of the properties of harmonic versus nonharmonic systems, and the presence or the absence of defects. In agreement with earlier studies, we found that in a purely harmonic (ballistic) thermal conductor (with spatially extended normal modes), there is no temperature gradient across the central part of the system. Whenever the system presents some form of thermal resistance (finite conductance) due to anharmonic effects, disorder, or extra random processes, a temperature gradient is present in the system. Furthermore a concrete example of such effects in a model of a one-dimension Al chain is provided in Appendix D.

We have also compared the results of the simulations using the GLE-2B approach to the results of other simulations that were carried out using standard thermostatting approaches (based on Markovian Langevin and Nosé-Hoover thermostats; see Fig. 11). In the latter cases, either a flat temperature profile or a temperature gradient across the central system can be obtained depending on the value used for the damping parameter. Upon the choice of this parameter, two different 
physical results can be obtained. Such a dilemma does not exist in the GLE-2B approach as it does not contain any adjustable parameters.

Furthermore, we have shown that the GLE-2B is able to treat, within the same scheme, two widely different transport regimes, i.e., systems which have ballistic (with no temperature gradient) or diffusive (with temperature gradient) thermal transport properties. This is a crucial point since the crossover between ballistic and diffusive transport regimes has been observed experimentally [22] in organic molecules of different lengths connecting two electrodes, after having been predicted theoretically [11].

Penultimately we would like to add that the GLE-2B has also another advantage over the more commonly used thermostatting approaches. This method has been derived explicitly in order to be able to treat inherently nonequilibrium properties which cannot be simulated (in principle) by the $\mathrm{NH}$ thermostats. Furthermore, we have already shown in Appendix D of Ref. [52] that we can derive the GLE dynamics with a colored noise which is not simply proportional to the memory kernel (as is the case for the classical limit of the equilibrium fluctuation-dissipation theorem). This means that quantum effects of the baths can be incorporated in the GLE dynamics. The importance of such effects has been considered in Refs. [14,114]. Finally, it should be noticed that our GLE-2B approach is also perfectly appropriate to study timedependent phenomena. Such interesting phenomena, which involve proper dynamical behavior of systems, will be the subject of future studies.

\section{ACKNOWLEDGMENTS}

We acknowledge financial support from the UK EPSRC, under Grant No.EP/J019259/1. H.N., C.D.L., and L.K. acknowledge the stimulating research environment provided by the EPSRC Centre for Doctoral Training in Cross-Disciplinary Approaches to Non-Equilibrium Systems (CANES, EP/L015854/1). A.G. would like to acknowledge the Department of Physics at King's College London for funding the summer internship which resulted in her contribution to this project. Finally, the authors thank one of the referees for a careful and critical analysis of our results and for suggestions that strengthened the value of the present work.

\section{APPENDIX A: VERLET-TYPE ALGORITHM FOR THE EXTENDED LANGEVIN DYNAMICS WITH TWO BATHS}

Following the Markovian equations derived in Sec. II B and prescriptions given in Ref. [52], we use the following algorithm for a single time step $\Delta t$.

The algorithm is derived, in a manner similar to the Verlet algorithm, from a different splitting and a Trotter-like decomposition of the total Liouvillian for the extended Langevin dynamics of the system DOFs, $r_{i \alpha}$, and the auxiliary DOFs, $s_{1,2}^{\left(k_{v}\right)}$, associated with the two independent baths $v=1,2$. Such a decomposition has been shown to provide a more appropriate description of the velocity correlation functions [50].
Algorithm:

(A) Randomize and propagate the aDOFs,

$s_{v, x}^{\left(k_{v}\right)} \leftarrow a_{k_{v}} s_{v, x}^{\left(k_{v}\right)}+b_{k_{v}} \xi_{v, x}^{\left(k_{v}\right)}$,

for all $k_{v}$ and $v=1,2$ and $x=1,2$.

(B) Calculate all $g_{i \alpha, b_{v}}\left(\left\{r_{i \alpha}\right\}\right)$ from the

derivatives of $f_{b_{v}}\left(\left\{r_{i \alpha}\right\}\right)$.

(C) Propagate the DOFs and aDOFs over $\Delta t / 2$,

$v_{i \alpha} \leftarrow v_{i \alpha}+\left(f_{i \alpha}+f_{i \alpha}^{\mathrm{pol}}+f_{i \alpha}^{p \mathrm{GLE}}\right) \frac{\Delta t}{2 m_{i}}$,

$s_{v, 2}^{\left(k_{v}\right)} \leftarrow s_{v, 2}^{\left(k_{v}\right)}-\omega_{k_{v}} s_{v, 1}^{\left(k_{v}\right)} \frac{\Delta t}{2}$.

(D) Propagate the DOFs over $\Delta t$,

$r_{i \alpha} \leftarrow r_{i \alpha}+v_{i \alpha} \Delta t$.

(E) Recalculate all $g_{i \alpha, b_{v}}\left(\left\{r_{i \alpha}\right\}\right)$ from the

derivatives of $f_{b_{v}}\left(\left\{r_{i \alpha}\right\}\right)$.

(F) Propagate the aDOFs over $\Delta t$,

$s_{v, 1}^{\left(k_{v}\right)} \leftarrow s_{v, 1}^{\left(k_{v}\right)}+\left(\omega_{k_{v}} s_{v, 2}^{\left(k_{v}\right)}+f_{k_{v}}^{s \mathrm{GLE}}\right) \Delta t$.

(G) Propagate the DOFs and aDOFs over $\Delta t / 2$,

$v_{i \alpha} \leftarrow v_{i \alpha}+\left(f_{i \alpha}+f_{i \alpha}^{\mathrm{pol}}+f_{i \alpha}^{p \mathrm{GLE}}\right) \frac{\Delta t}{2 m_{i}}$,

$s_{v, 2}^{\left(k_{v}\right)} \leftarrow s_{v, 2}^{\left(k_{\nu}\right)}-\omega_{k_{v}} s_{\nu, 1}^{\left(k_{v}\right)} \frac{\Delta t}{2}$.

(H) Randomize and propagate all the aDOFs,

$$
s_{v, x}^{\left(k_{\nu}\right)} \leftarrow a_{k_{v}} s_{v, x}^{\left(k_{\nu}\right)}+b_{k_{v}} \xi_{v, x}^{\left(k_{\nu}\right)} \text {. }
$$

The different forces, $f_{i \alpha}, f_{i \alpha}^{\mathrm{pol}}, f_{i \alpha}^{p \mathrm{GLE}}, f_{k_{\nu}}^{s \mathrm{GLE}}$, are explained below. The force

$$
f_{i \alpha}=-\frac{\partial V(\mathbf{r})}{\partial r_{i \alpha}}
$$

is the force acting on the system DOFs $i \alpha$ due to the interaction between the atoms in the system and in the bath region(s); the "polaronic" force $f_{i \alpha}^{\mathrm{pol}}$

$$
\begin{aligned}
f_{i \alpha}^{\mathrm{pol}} & =\sum_{\nu} \sum_{b_{v}, b_{v}^{\prime}} \sqrt{\mu_{l_{v}} \mu_{l_{v}^{\prime}}} g_{i \alpha, b_{v}} \Pi_{b_{v} b_{v}^{\prime}}(0) f_{b_{v}^{\prime}} \\
& =\sum_{\nu} \sum_{b_{v}, b_{v}^{\prime}, k_{v}} \sqrt{\mu_{l_{v}} \mu_{l_{v}^{\prime}}} g_{i \alpha, b_{v}} c_{b_{v}}^{\left(k_{v}\right)} c_{b_{v}}^{\left(k_{v}\right)} f_{b_{v}^{\prime}}
\end{aligned}
$$

(with $b_{v} \equiv l_{\nu} \gamma$ for DOFs the $v$ th bath) is the force acting on the system DOFs $i \alpha$ due to the interaction between the system and bath regions which induces a displacement of the positions of the harmonic oscillators characterizing the baths. In Eq. (A3), we used the fact that $\Pi_{b_{v} b_{v}^{\prime}}(0)$ is the inverse Fourier transform (evaluated at $\tau=0$ ) of $\Pi_{b_{v} b_{v}^{\prime}}(\omega)$ given by Eq. (18).

The force $f_{i \alpha}^{p \mathrm{GLE}}$ acts on the system DOFs $i \alpha$ and arises from the generalized Langevin equations:

$$
f_{i \alpha}^{p \mathrm{GLE}}=\sum_{\nu} \sum_{b_{v}, k_{v}} \sqrt{\frac{\mu_{l_{v}}}{\bar{\mu}_{\nu}}} g_{i \alpha, b_{v}}\left(\left\{r_{i \alpha}\right\}\right) c_{b_{v}}^{\left(k_{\nu}\right)} s_{1}^{\left(k_{v}\right)} .
$$


The force $f_{k_{\nu}}^{s \mathrm{GLE}}$ acts on the aDOFs $s_{1}^{\left(k_{\nu}\right)}$ and also arises from the generalized Langevin equations

$$
f_{k_{v}}^{s \mathrm{GLE}}=-\sum_{i \alpha, b_{v}} \sqrt{\mu_{l_{v}} \bar{\mu}_{v}} g_{i \alpha, b_{v}}\left(\left\{r_{i \alpha}\right\}\right) c_{b_{v}}^{\left(k_{v}\right)} v_{i \alpha} .
$$

The integration of the dissipative part of the dynamics of the aDOFs [see steps (A) and (F) in the algorithm] includes the coefficients $a_{k_{v}}=\exp \left(-\Delta t / 2 \tau_{k_{v}}\right)$ and $b_{k_{v}}=$ $\left[k_{B} T_{v} \bar{\mu}_{v}\left(1-a_{k_{v}}^{2}\right)\right]^{1 / 2}$ and the uncorrelated random variable $\xi_{1,2}^{\left(k_{v}\right)}$ corresponding to the white noise.

\section{APPENDIX B: EVOLUTION AND STATISTICS OF THE ENERGY}

In this appendix we show the time evolution of the kinetic energy and potential energy for a system under nonequilibrium conditions. We also calculate the statistical distribution of the kinetic energy and briefly discuss the convergence of the numerical calculations for the GLE-2B and LG approaches.

Figure 12 shows the time evolution of the total kinetic energy $E_{\mathrm{kin}}^{\mathrm{TOT}}$ and of the total potential energy $E_{\mathrm{pot}}^{\mathrm{TOT}}$ for the nonequilibrium conditions $T_{L}=150 \mathrm{~K}$ and $T_{R}=50 \mathrm{~K}$. This is a typical result, and the following discussion can be applied (qualitatively) to other combinations of bath temperatures [115]. For all calculations, $E_{\mathrm{kin}}^{\mathrm{TOT}}$ reaches an asymptotic (nonequilibrium) stationary value. For the GLE-2B calculations, the stationary regime is obtained after $80 \mathrm{ps}$ (around 40000 time steps), while for the LG calculations, the number of time steps needed to reach the stationary regime is strongly dependent on the value used for the damping parameter $\tau_{\text {damp }}$.

The asymptotic value of $E_{\mathrm{kin}}^{\mathrm{TOT}}$ is around $0.8 \mathrm{eV}$, which is completely different than the corresponding values expected from equilibrium and the equipartition principle for the two bath temperatures, i.e., $\frac{3}{2} N k T_{L}=0.38 \mathrm{eV}$ and $\frac{3}{2} N k T_{R}=1.14$ $\mathrm{eV}$. This is indeed not surprising as the central system is not in an equilibrium state.

Interestingly, for the nonequilibrium conditions, the choice of the value of the damping parameter $\tau_{\text {damp }}$ for the LG calculations is crucial to obtain the proper physics. One cannot simply use the best $\tau_{\text {damp }}$ to reproduce the time evolution of the kinetic energy, as done for the equilibrium case [53].

The influence of the value of $\tau_{\text {damp }}$ is reflected in the distribution of the kinetic energy shown in Fig. 13. Only the LG calculations performed with a large value of $\tau_{\text {damp }}$ reproduce the distribution of $E_{\text {kin }}^{\text {TOT }}$ obtained from the GLE-2B approach. The LG calculations performed with $\tau_{\text {damp }}=7$ ps produce a much broader distribution.

Furthermore, in order to obtain the correct temperature profile given by the GLE-2B approach, one needs to use a large $\tau_{\text {damp }}$ in the LG approach. The LG calculations made with $\tau_{\text {damp }}=7 \mathrm{ps}$, which result in a gradient in the temperature profile (i.e., a completely different physics behavior; see Fig. 8), show a time evolution of $E_{\text {kin }}^{\mathrm{TOT}}$ that is much more similar to that obtained from the GLE-2B method. These results confirm that the choice of the adjustable parameter for the LG method is crucial for being able to simulate the proper physical behavior. This is also true for the $\mathrm{NH}$ thermostatting approach (results not shown).

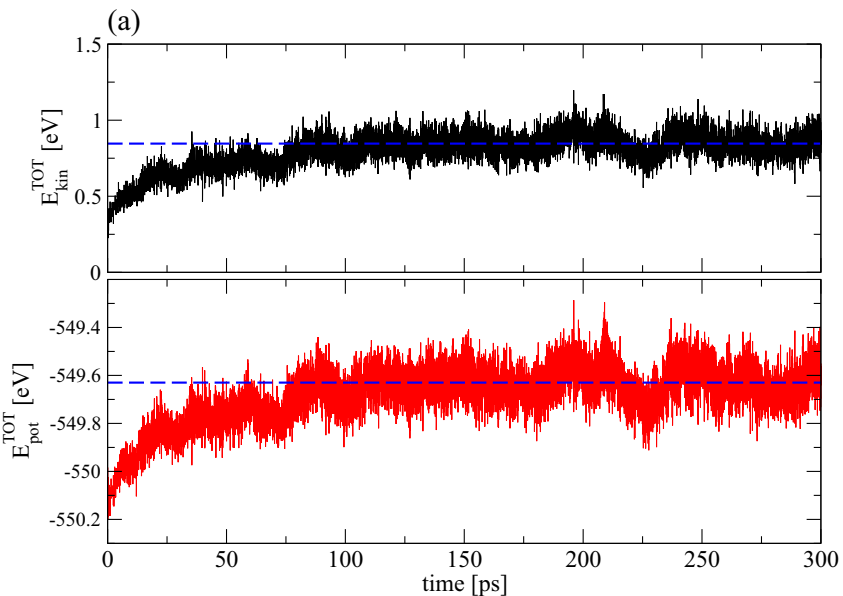

(b)

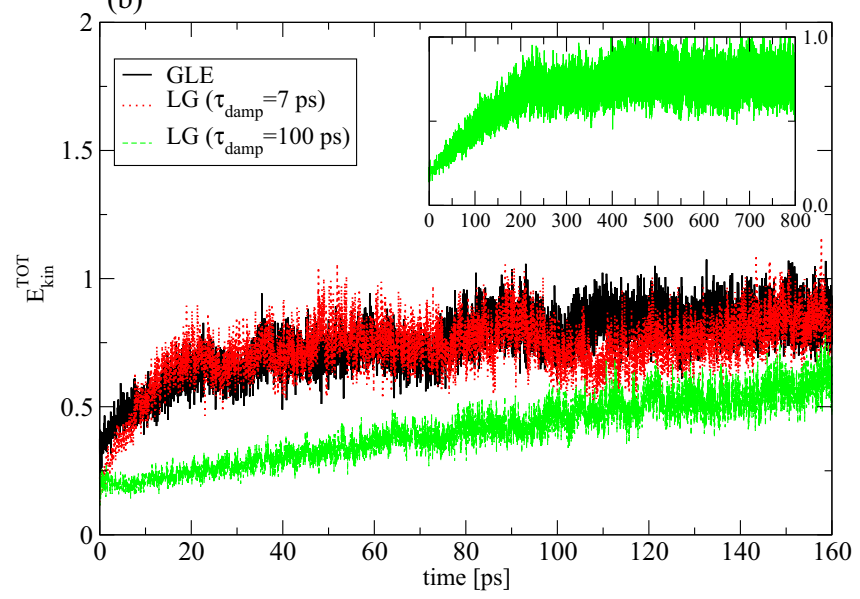

FIG. 12. (a) Evolution of the total kinetic energy $E_{\text {kin }}^{\text {TOT }}$ and of the total potential energy $E_{\text {pot }}^{\mathrm{TOT}}$ of the system shown in Fig. 2 versus time ( $\Delta t=2 \mathrm{fs}$ ) [115]. The calculations are performed with the bath temperatures $T_{L}=150, T_{R}=50$. The horizontal dashed blue lines correspond to the averages of the energy over the time range 100 to $300 \mathrm{ps}$. The convergence is fairly well obtained after $\sim 80 \mathrm{ps}$ (40 000 time steps). (b) Evolution of the total kinetic energy $E_{\text {kin }}^{\mathrm{TOT}}$ of the central system only (59 atoms from layers L3 to R3). For the LG calculations, the convergence towards the stationary value occurs after roughly $80 \mathrm{ps}$ for the damping time $\tau_{\text {damp }}=7 \mathrm{ps}$. The stationary state is reached after more time steps, as expected, for the larger damping time $\tau_{\text {damp }}=100 \mathrm{ps}$ (see inset where it is apparent that the stationary value is reached after 500 ps or 250000 time steps).

\section{APPENDIX C: INFLUENCE OF THE COUPLING TO THE BATHS AND THE SYSTEM SIZE}

In this section, we consider another way of coupling the $\mathrm{Al}$ nanowire to the thermal baths. We treat a system similar to the system shown in Fig. 2 and Fig. 3 by including in the central system only the 7 layers labeled A to $\mathrm{G}$ (system containing 31 atoms). The atoms of the layers L1, L2, and L3 (R1, R2, and R3) have now been incorporated in the $\mathrm{L}(\mathrm{R})$ bath regions themselves.

For this system, we obtain the same physical results for the temperature profile as those presented in the main text [116]. For low temperatures and small $\Delta T=T_{L}-T_{R}$, the central 


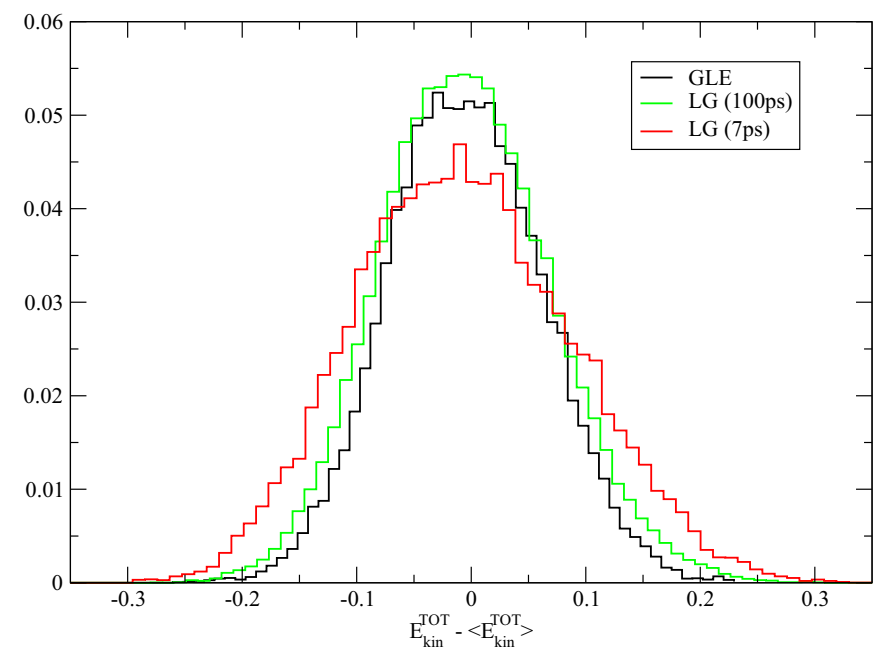

FIG. 13. Histograms of the total kinetic energy of the central system containing 59 atoms for the bath temperatures $T_{L}=150$, $T_{R}=50$. The averaged kinetic energy $\left\langle E_{\text {kin }}^{\mathrm{TOT}}\right\rangle \sim 0.8 \mathrm{eV}$ is the energy reference.

system is harmonic and no temperature gradient is obtained across the system; see Fig. 14.

Once more, the LG thermostats provide two different temperature profiles depending up the value of the damping parameter $\tau_{\text {damp }}$. For small values of the damping parameter $\left(\tau_{\text {damp }}=7 \mathrm{ps}\right)$, we obtain a small temperature gradient in the central system while for larger values of the damping parameter $\tau_{\text {damp }}=100 \mathrm{ps}$, the LG thermostats provide a flat temperature profile.

In terms of convergence versus the bath size, one should first note that, in the GLE-2B approach, we do not choose which group of atoms is connected to a thermal bath, as this is usually performed when using more conventional LG or NH thermostats.

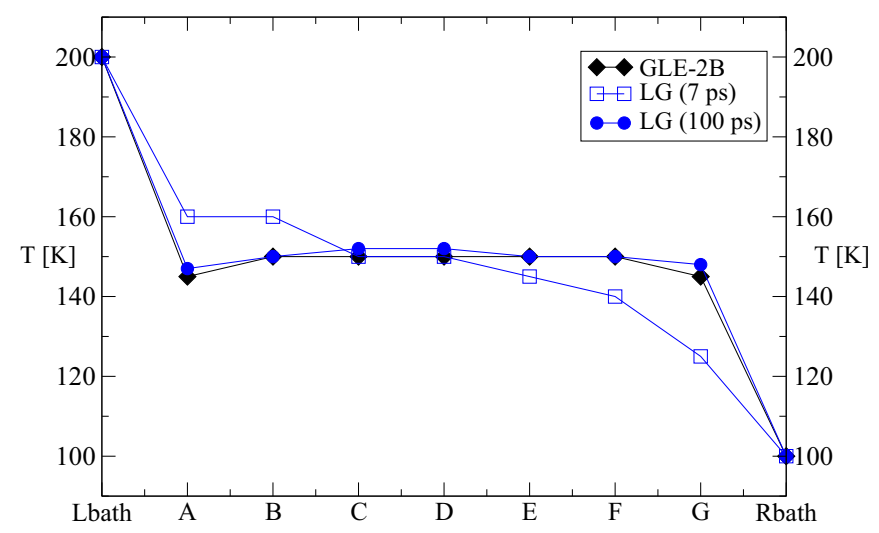

FIG. 14. Temperature profiles across the system consisting only of the 7 layers labeled $A$ to $G$ in Figs. 3 and 11. The different bath temperatures are $T_{L}=200, T_{R}=100$. While the GLE-2B calculations provide a uniform temperature profile inside the system, the LG thermostats show either the building up of a temperature gradient in the central system $\left(\tau_{\text {damp }}=7 \mathrm{ps}\right)$ or a flat temperature profile $\left(\tau_{\mathrm{damp}}=100 \mathrm{ps}\right)$.
The coupling of the central system to the thermal bath $v$ is obtained by the coupling of the DOFs $i \alpha$ to the aDOFs $s_{v, 1}^{\left(k_{v}\right)}$ via the matrix elements $g_{i \alpha, b_{v}}$; see Eq. (19) and Eq. (14). Such a coupling exists only when the matrix element $g_{i \alpha, b_{v}}$ is nonzero. We recall that $g_{i \alpha, b_{v}}(\mathbf{r})=\partial f_{b_{v}}(\mathbf{r}) / \partial r_{i \alpha}$ is the derivative, with respect to the coordinate of the DOFs $i \alpha$, of the force $f_{b_{v}}$ felt by the atom $l_{v}$ of the bath $v$ with DOFs $b_{v} \equiv l_{\nu} \gamma$.

The range of the matrix elements $g_{i \alpha, b_{v}}$ is determined by the cutoff of the interatomic potential used in the calculations. The range of the quantities $g_{i \alpha, b_{v}}$ is actually smaller than the cutoff of the interatomic potential, as the former is the second derivative (versus spatial coordinates) of the latter.

Hence there is no need to increase the size of the reduced bath region, as long as DOFs of the central system are properly coupled to the existing atoms in the reduced bath region. Indeed, adding atoms in the reduced bath region (which corresponds to $g_{i \alpha, b_{v}}=0$ elements) will not change the dissipative dynamics of the atoms of the central region.

However, one should not forget that the infinite spatial extension of the baths has already been taken into account. In particular, the continuous vibrational spectra of the infinite baths has been obtained through the calculation of their dynamical matrix and subsequently in the mapping procedure described in Secs. II B and IID (for more details, see also Ref. [53]).

The coupling to the thermal baths comes directly from the construction of the geometry of the system itself and from the cutoff of the interatomic potential. It is not controlled by the user as usually done with LG or NH thermostats. This is, once more, one of the main differences between the consistent (and more elaborate) GLE-2B approach and the main-stream LG or NH thermostatting approach.

As far as the convergence versus the size of the central system is concerned, we have already mentioned in the main text that nonballistic transport properties can be obtained for much longer and heterogeneous systems [78,79,81-87,90,9295,97-112]. The presence of long-wavelength acoustic modes and their indirect coupling (via the baths) with other delocalized vibrational modes can also lead to the establishment of a more diffusive transport property associated with the presence of a temperature profile in the central system. The study of the transport properties versus the size of the central system is important, but out of the scope of the present paper, and will be treated elsewhere.

\section{APPENDIX D: A ONE-DIMENSIONAL TOY MODEL}

In this section, we consider a toy model for a onedimensional system: a chain made of $11 \mathrm{Al}$ atoms connected to two baths as shown in Fig. 15. This is a simpler system than the three-dimensional wire considered in the main body of the paper. We use the GLE-2B approach for the dynamics of the central chain and study the local temperature profile of the perfect chain and of the chains containing one or two defects. To model the defect, we simply change the mass of the corresponding atom while conserving the same embedded atom method (EAM) interatomic potential for all atoms in the GLE-2B calculations.

To make the system simpler, we constrain the dynamics of the atoms in the central chain to a purely one-dimensional 


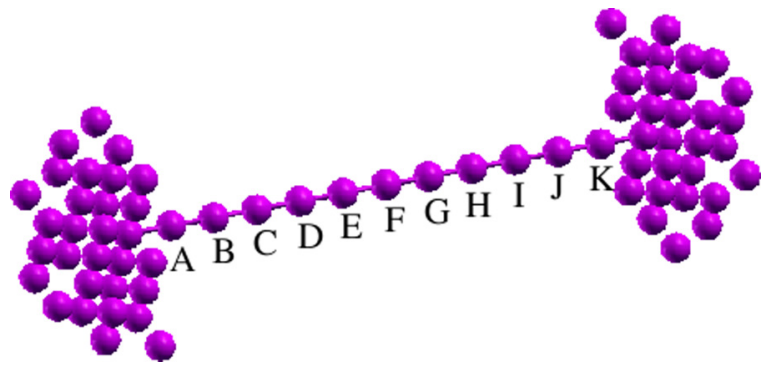

FIG. 15. Model for a one-dimensional Al chain (central system containing 11 atoms labeled A to K) connected to the left $(L)$ and right $(R)$ baths. For the GLE calculations, the $L$ and $R$ bath reduced region contains 30 atoms each. For the calculations of the dynamical matrix and the mapping of the polarization matrix, larger baths (containing each 95 atoms) were considered. The mapping of the polarization matrix elements is done using $78 \mathrm{aDOFs}$ with a set of 78 parameters $\left\{c_{b_{v}}^{\left(k_{\nu}\right)}, \tau_{k_{v}}, \omega_{k_{v}}\right\}$ for each bath $v=L, R$.

problem; i.e., the atoms can move only along the main axis (the $x$ axis) of the chain. Therefore each atom in the central region is associated with only one degree of freedom.

For a system at equilibrium, $T_{L}=T_{R}$, the GLE-2B calculations provide a velocity distribution for each atom of the central region which follows, as expected, the statistical Gaussian distribution of a single degree of freedom given by

$$
f_{1 \mathrm{D}}\left(v_{x}\right)=\sqrt{\frac{m}{2 \pi k T}} \exp \left(-\frac{m v_{x}^{2}}{2 k T}\right) .
$$

For the nonequilibrium conditions, $T_{L} \neq T_{R}$, the velocity distributions, calculated from the GLE-2B, for the atoms of the central chain also follow the line shape of a Gaussian distribution. However the associated temperature varies across the chain.

Figure 16 shows the temperature profile across the chain when the masses of all atoms in the central chain are equal. For "low" temperatures $T_{L, R}$, the homogeneous system behaves as a harmonic system and one obtains a flat temperature profile as expected for integrable systems. For "higher" temperatures, the motion of some of the atoms starts to sample the anharmonic part of the interatomic potential. Therefore some parts of the system are not harmonic anymore and a temperature gradient starts to build up.

Note that because the dynamics of the system is strongly constrained, no structural instability is possible and higher local effective temperatures (in comparison with the $3 \mathrm{D}$ short wire considered in the main part of the paper) can be investigated in order to achieve the anharmonic regime.

Figure 17 shows the temperature profile across the chain when one introduces a localized defect in the chain. Calculations are performed when the mass of the atom labeled $\mathrm{H}$ in the chain is increased by $10 \%$ to $40 \%$ and when the mass of both atoms $\mathrm{C}$ and $\mathrm{H}$ is increased by $40 \%$. One can see that the introduction of a defect in the chain (in the harmonic regime) leads to the buildup of a temperature gradient. Such an effect is clearly obtained for a mass increase larger than $10 \%$ and in the cases of more than one defect present in the chain.

We have checked that, in the presence of defects, the vibration modes of the chain are slightly more localized

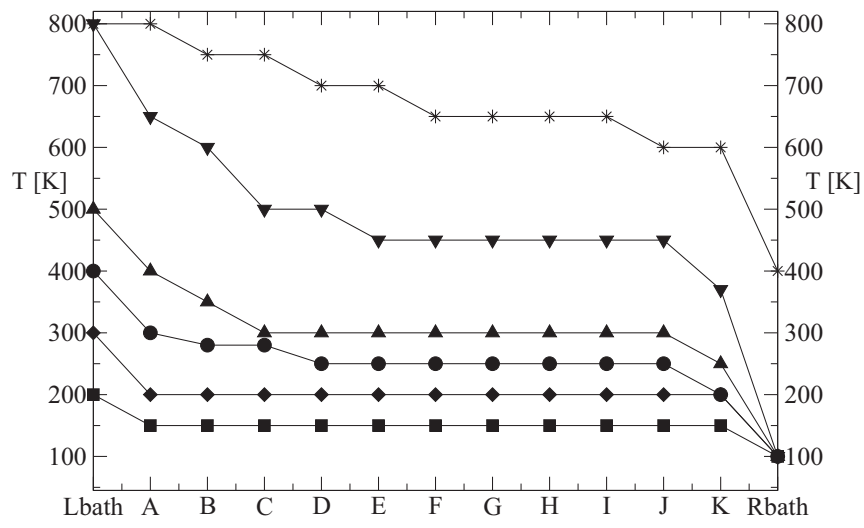

FIG. 16. Temperature profiles across the central 1D chain for different bath temperatures $T_{L, R}$. The labels Lbath (Rbath) represent the temperatures at the left (right) bath. The other labels A to $\mathrm{K}$ indicate different atoms in the central chain. For "low" temperatures $T_{L, R}$, the system is harmonic and one gets a flat temperature profile as expected for integrable systems. For "higher" temperatures, the motion of some of the atoms samples the anharmonic part of the interatomic potential, and therefore the system is nonintegrable and a temperature gradient is built up.

(around the defects) than for the perfect chain. Furthermore the amplitudes of the vibration modes at the ends of the chain are also different in the presence of defects in the chain. Such effects are thought to hinder the heat transport from one bath to the other and hence lead to the absence of a flat temperature profile across the chain.

Thus, the calculations shown here for a toy model of a one-dimensional Al chain present the same qualitative physics as that obtained for the three-dimensional short wire described in the main text.

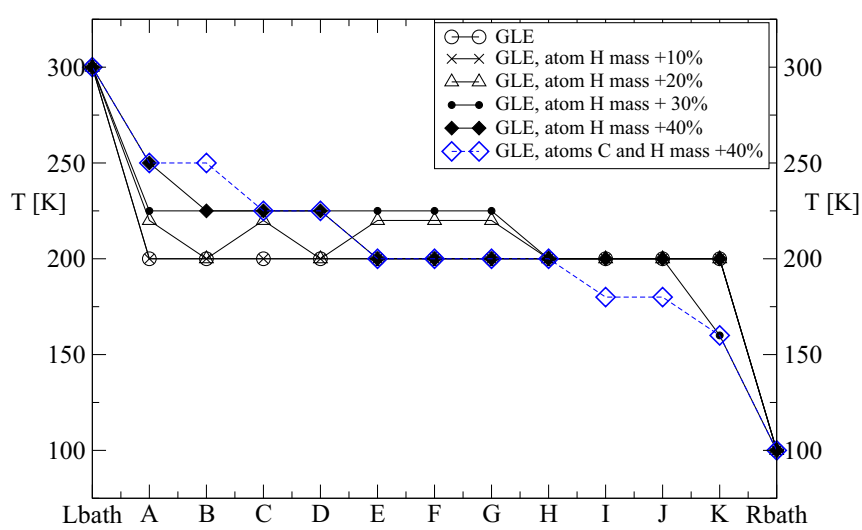

FIG. 17. Temperature profiles across the central 1D chain for different defects present in the chain. The labels Lbath (Rbath) represent the temperatures at the left (right) bath. The other labels A to $\mathrm{K}$ indicate different atoms in the central chain. The mass of atom $\mathrm{H}$ in Fig. 15 is increased by $10 \%, 20 \%, 30 \%$, or $40 \%$. The case of two defects (atoms $\mathrm{C}$ and $\mathrm{H}$ with their mass increased by $40 \%$ ) is also shown. For the temperatures considered, the chain is in the harmonic regime. However the presence of defects, and their associated more localized vibration modes, leads to the build up of a temperature gradient across the chain. 
[1] S. Berber, Y.-K. Kwon, and D. Tománek, Phys. Rev. Lett. 84, 4613 (2000).

[2] P. Kim, L. Shi, A. Majumdar, and P. L. McEuen, Phys. Rev. Lett. 87, 215502 (2001)

[3] L. Shi and A. Majumdar, J. Heat Transfer 124, 329 (2002).

[4] C. W. Padgett and D. W. Brenner, Nano Lett. 4, 1051 (2004).

[5] M. Hu, P. Keblinski, J.-S. Wang, and N. Raravikar, J. Appl. Phys. 104, 083503 (2008).

[6] C. W. Padgett, O. Shenderova, and D. W. Brenner, Nano Lett. 6, 1827 (2006).

[7] N. Yang, G. Zhang, and B. Li, Nano Lett. 8, 276 (2008).

[8] S. K. Estreicher and T. M. Gibbons, Physica B 404, 4509 (2009).

[9] A. Majumdar, Annu. Rev. Mater. Sci. 29, 505 (1999).

[10] D. Segal and A. Nitzan, J. Chem. Phys. 117, 3915 (2002).

[11] D. Segal, A. Nitzan, and P. Hänggi, J. Chem. Phys. 119, 6840 (2003).

[12] N. Mingo and Liu Yang, Phys. Rev. B 68, 245406 (2003).

[13] Z. Yao, J.-S. Wang, B. Li, and G.-R. Liu, Phys. Rev. B 71, 085417 (2005).

[14] J.-S. Wang, Phys. Rev. Lett. 99, 160601 (2007).

[15] Y. Dubi and M. Di Ventra, Rev. Mod. Phys. 83, 131 (2011).

[16] J. R. Widawsky, P. Darancet, J. B. Neaton, and L. Venkataraman, Nano Lett. 12, 354 (2012).

[17] D. G. Cahill, K. Goodson, and A. Majumdar, J. Heat Transfer 124, 223 (2002).

[18] E. Pop, Nano. Res. 3, 147 (2010).

[19] M. Zebarjadi, K. Esfarjani, M. S. Dresselhaus, Z. F. Ren, and G. Chen, Energy Environ. Sci. 5, 5147 (2012).

[20] B. Gotsmann and M. A. Lantz, Nat. Mater. 12, 59 (2013).

[21] F. Menges, H. Riel, A. Stemmer, C. Dimitrakopoulos, and B. Gotsmann, Phys. Rev. Lett. 111, 205901 (2013).

[22] T. Meier, F. Menges, P. Nirmalraj, H. Hölscher, H. Riel, and B. Gotsmann, Phys. Rev. Lett. 113, 060801 (2014).

[23] J. Li, D.-Q. Zheng, and W.-R. Zhong, Europhys. Lett. 112, 24006 (2015).

[24] H. Mori, Prog. Theor. Phys. 33, 423 (1965).

[25] S. A. Adelman and J. Doll, J. Chem. Phys. 64, 2375 (1976).

[26] S. A. Adelman, J. Chem. Phys. 73, 3145 (1980).

[27] D. L. Ermak and H. Buckholz, J. Comput. Phys. 35, 169 (1980).

[28] B. Carmeli and A. Nitzan, Chem. Phys. Lett. 102, 517 (1983).

[29] E. Cortés, B. J. West, and K. Lindenberg, J. Chem. Phys. 82, 2708 (1985).

[30] K. Lindenberg and B. J. West, The Nonequilibrium Statistical Mechanics of Open and Closed Systems (Wiley-VCH, New York, 1990).

[31] R. Tsekov and E. Ruckenstein, J. Chem. Phys. 100, 1450 (1994).

[32] R. Tsekov and E. Ruckenstein, J. Chem. Phys. 101, 7844 (1994).

[33] H. Risken, The Fokker-Planck Equation: Methods of Solutions and Applications, 2nd ed. (Springer, Berlin, 1996).

[34] R. Hernandez, J. Chem. Phys. 111, 7701 (1999).

[35] R. Zwanzig, Nonequilibrium Statistical Mechanics (Oxford University Press, Oxford, UK, 2001).

[36] R. Kupferman, J. Stat. Phys. 114, 291 (2004).

[37] J.-D. Bao, J. Stat. Phys. 114, 503 (2004).

[38] J. Łuczka, Chaos 15, 026107 (2005).
[39] S. Izvekov and G. A. Voth, J. Chem. Phys. 125, 151101 (2006).

[40] I. Snook, The Langevin and Generalised Langevin Approach to the Dynamics of Atomic, Polymeric and Colloidal Systems (Elsevier, Amsterdam, 2007).

[41] C. M. van Vliet, Equilibrium and Non-Equilibrium Statistical Mechanics (World Scientific, Singapore, 2008).

[42] L. Kantorovich, Phys. Rev. B 78, 094304 (2008).

[43] M. Ceriotti, G. Bussi, and M. Parrinello, Phys. Rev. Lett. 102, 020601 (2009).

[44] M. Ceriotti, G. Bussi, and M. Parrinello, J. Chem. Theory Comput. 6, 1170 (2010).

[45] P. Siegle, I. Goychuk, P. Talkner, and Peter Hänggi, Phys. Rev. E 81, 011136 (2010).

[46] S. Kawai and T. Komatsuzaki, J. Chem. Phys. 134, 114523 (2011).

[47] J. A. Morrone, T. E. Markland, M. Ceriotti, and B. J. Berne, J. Chem. Phys. 134, 014103 (2011).

[48] M. Ceriotti, D. E. Manolopoulos, and M. Parrinello, J. Chem. Phys. 134, 084104 (2011).

[49] D. Pagel, A. Alvermann, and H. Fehske, Phys. Rev. E 87, 012127 (2013).

[50] B. Leimkuhler and C. Matthews, J. Chem. Phys. 138, 174102 (2013).

[51] A. D. Baczewski and S. D. Bond, J. Chem. Phys. 139, 044107 (2013).

[52] L. Stella, C. D. Lorenz, and L. Kantorovich, Phys. Rev. B 89, 134303 (2014).

[53] H. Ness, L. Stella, C. D. Lorenz, and L. Kantorovich, Phys. Rev. B 91, 014301 (2015).

[54] H. C. Andersen, J. Chem. Phys. 72, 2384 (1980).

[55] S. Nosé, Mol. Phys. 52, 255 (1984).

[56] S. Nosé, J. Chem. Phys. 81, 511 (1984).

[57] W. G. Hoover, Phys. Rev. A 31, 1695 (1985).

[58] D. Toton, C. D. Lorenz, N. Rompotis, N. Martsinovich, and L. Kantorovich, J. Phys. Condens. Matter 22, 074205 (2010).

[59] L. Kantorovich and N. Rompotis, Phys. Rev. B 78, 094305 (2008).

[60] S. J. Plimpton, J. Comput. Phys. 117, 1 (1995).

[61] D. T. Gillespie, Am. J. Phys. 64, 1246 (1996).

[62] D. T. Gillespie, Am. J. Phys. 64, 225 (1996).

[63] There exist different possible choices for the Trotter factorization of the Liouvillian. From our understanding of Ref. [50], the Trotter decomposition we use to obtain our GLE-MD algorithm has been shown to provide a more appropriate description of the velocity correlation functions. An accurate statistical description of the velocities is crucial in the present paper, as we extract the local temperature from the velocities' statistical distributions.

[64] It should noted that there cannot be any form of coupling between the two baths. Otherwise they would not be independent and therefore it would not be possible to consider them as being kept at their own thermodynamic equilibrium characterized by the temperature $T_{v}$.

[65] M. Ferrario and P. Grigolini, J. Math. Phys. 20, 2567 (1979).

[66] F. Marchesoni and P. Grigolini, J. Chem. Phys. 78, 6287 (1983).

[67] C. C. Wan, J.-L. Mozos, J. Wang, and H. Guo, Phys. Rev. B 55, R13393(R) (1997). 
[68] G. Taraschi, J.-L. Mozos, C. C. Wan, H. Guo, and J. Wang, Phys. Rev. B 58, 13138 (1998).

[69] J. Taylor, H. Guo, and J. Wang, Phys. Rev. B 63, 245407 (2001).

[70] M. S. Daw and M. I. Baskes, Phys. Rev. B 29, 6443 (1984).

[71] We have used the data file A199.eam.alloy from the NIST Interatomic Potential Repository Project (http://www.ctcms.nist.gov/potentials) as provided by Ref. [72].

[72] Y. Mishin, D. Farkas, M. J. Mehl, and D. A. Papaconstantopoulos, Phys. Rev. B 59, 3393 (1999).

[73] The velocity distributions are calculated from the averages of several realizations of each velocity distribution obtained at a given time step, after the system is thermalized. For $T=200 \mathrm{~K}$, the thermalization is reached after 78 ps of GLE dynamics, i.e., after 40000 time steps with $\Delta t=2$ fs. The distributions are averaged over all the time steps in the time range $t=[78,144]$ ps.

[74] Calculation using the LG thermostats and the GLE-2B approach were performed at equilibrium when the system is uniformly thermostatted at $T_{L}=T_{R}$. From the results of these calculations, we observed that the Al nanowire melts down at $T=500 \mathrm{~K}$ for the EAM interatomic potential we used. During the MD run, we observe the formation of the smaller constriction in the middle of the nanowire and eventually all the atoms of the layers A to $\mathrm{F}$ end up on the surfaces of the $\mathrm{L}$ and $\mathrm{R}$ bath regions. At temperatures $T=400-450$, we also obtain the formation of a smaller constriction in the middle of the nanowire (with atoms moving towards the left and right sides of the constriction). The constriction consists of 3 layers of only 2 atoms in each layer. This is what we call "structural instabilities," meaning that the central system does not have the same atomic configuration (layers made of 4 or 5 atoms). Such strong modifications of the configuration of the system make it difficult to perform a consistent analysis of the (layer-by-layer) temperature profiles. One should notice that the melting of $\mathrm{Al}$ surfaces, depending on the Miller indices $(h k l)$, may occur even at temperatures which are 150 to $200 \mathrm{~K}$ below the bulk melting temperature [see for example P. Stoltze, J. K. Nørskov, and U. Landman, Phys. Rev. Lett. 61, 440 (1988)], i.e., roughly around $730-780 \mathrm{~K}$. The coordination of an $\mathrm{Al}$ atom at the surface is roughly half of that of an atom in the bulk. Therefore we believe that it is not surprising that the melting of the nanowire happens at even lower temperature as the coordination of the atoms inside the nanowire is even smaller that the coordination of surface atoms.

[75] J. P. Small, L. Shi, and P. Kim, Solid State Commun. 127, 181 (2003).

[76] The GLE-2B runs are performed for 300 ps (150 000 time steps with $\Delta t=2 \mathrm{fs}$ ). The velocity histograms are obtained from statistical averaging over the time range 200 to $280 \mathrm{ps}$.

[77] Z. Rieder, J. L. Lebowitz, and E. Lieb, J. Math. Phys. 8, 1073 (1967).

[78] M. Rich and W. M. Visscher, Phys. Rev. B 11, 2164 (1975).

[79] H. Spohn and J. L. Lebowitz, Commun. Math. Phys. 54, 97 (1977).

[80] G. Casati, Nuovo Cimento 52, 257 (1979).

[81] B. Hu, B. Li, and H. Zhao, Phys. Rev. E 61, 3828 (2000).

[82] D. Segal, J. Chem. Phys. 128, 224710 (2008).
[83] V. Kannan, A. Dhar, and J. L. Lebowitz, Phys. Rev. E 85, 041118 (2012).

[84] K. Sääskilahti, J. Oksanen, R. P. Linna and J. Tulkki, Phys. Rev. E 86, 031107 (2012).

[85] G. T. Landi and M. J. de Oliveira, Phys. Rev. E 87, 052126 (2013).

[86] U. Zürcher and P. Talkner, Phys. Rev. A 42, 3278 (1990).

[87] K. Saito, S. Takesue, and S. Miyashita, Phys. Rev. E 54, 2404 (1996).

[88] K. Saito, S. Takesue, and S. Miyashita, Phys. Rev. E 61, 2397 (2000).

[89] A. Dhar and B. Sriram Shastry, Phys. Rev. B 67, 195405 (2003).

[90] C. Gaul and H. Büttner, Phys. Rev. E 76, 011111 (2007).

[91] A. Asadian, D. Manzano, M. Tiersch, and H. J. Briegel, Phys. Rev. E 87, 012109 (2013).

[92] E. A. Jackson, J. R. Pasta, and J. F. Waters, J. Comput. Phys. 2, 207 (1968).

[93] R. J. Rubin and W. L. Greer, J. Math. Phys. 12, 1686 (1971).

[94] M. Bolsterli, M. Rich, and W. M. Visscher, Phys. Rev. A 1, 1086 (1970).

[95] H. Nakazawa, Prog. Theor. Phys. Suppl. 45, 231 (1970).

[96] A. M. Stoneham, Theory of Defects in Solids: Electronic Structure of Defects in Insulators and Semiconductors (Oxford University Press, Oxford, 1975), Chap. 11.

[97] J.-P. Eckmann, C.-A. Pillet, and L. Rey-Bellet, Commun. Math. Phys. 201, 657 (1999).

[98] T. Hatano, Phys. Rev. E 59, R1 (1999).

[99] Y. Zhang and H. Zhao, Phys. Rev. E 66, 026106 (2002).

[100] E. Pereira and R. Falcao, Phys. Rev. E 70, 046105 (2004).

[101] T. Mai and O. Narayan, Phys. Rev. E 73, 061202 (2006).

[102] J. Bricmont and A. Kupiainen, Commun. Math. Phys. 274, 555 (2007).

[103] T. Hu and Y. Tang, J. Phys. Soc. Jpn. 79, 064601 (2010).

[104] C. Giberti and L. Rondoni, Phys. Rev. E 83, 041115 (2011).

[105] E. Pereira, Physica A 390, 4131 (2011).

[106] T. N. Shah and P. N. Gajjar, Commun. Theor. Phys. 59, 361 (2013).

[107] G. P. Tsironis, A. R. Bishop, A. V. Savin, and A. V. Zolotaryuk, Phys. Rev. E 60, 6610 (1999).

[108] C. Kipnis, C. Marchioro, and E. Presutti, J. Stat. Phys. 27, 65 (1982).

[109] C. Bernardin and S. Olla, J. Stat. Phys. 121, 271 (2005).

[110] S. Lepri, C. Mejía-Monasterio, and A. Politi, J. Phys. A: Math. Theor. 42, 025001 (2009).

[111] C. Bernardin, V. Kannan, J. L. Lebowitz, and J. Lukkarinen, J. Stat. Phys. 146, 800 (2012).

[112] E. B. Davis, J. Stat. Phys. 18, 161 (1978).

[113] J.-S. Wang and B. Li, Phys. Rev. E 70, 021204 (2004).

[114] M. Ceriotti, G. Bussi, and M. Parrinello, Phys. Rev. Lett. 103, 030603 (2009).

[115] The atoms of the bath reduced regions are present in the GLE2B method (implemented in the LAMMPS code), as one needs to calculate the matrix elements $g_{i \alpha, b_{v}}(\mathbf{r})$ for the instantaneous atomic positions of the central system. However the atoms in the bath reduced regions are kept fixed during the GLE-2B runs; hence they do not contribute to the total kinetic energy 
of the system shown in Fig. 2. Furthermore the contribution to the total potential energy from these atoms is just a constant throughout the GLE-2B runs.

[116] The GLE-2B runs, as well as the LG runs with $\tau_{\text {damp }}=7 \mathrm{ps,}$ are performed for $120 \mathrm{ps}$ (120000 time steps with $\Delta t=1$ fs). The steady state is reached around $80 \mathrm{ps}$. The velocity histograms (from which the local temperature is extracted) are obtained from statistical averaging over the time range 100 to $120 \mathrm{ps}$. For the LG runs with $\tau_{\text {damp }}=100 \mathrm{ps}$, the steady state is reached after a longer time, $\sim 300 \mathrm{ps}$. The velocity histograms are obtained from statistical averaging over the time range 450 to $600 \mathrm{ps}$. 